\title{
MULTITYPE BRANCHING BROWNIAN MOTION AND TRAVELING WAVES
}

\author{
YAN-XIA REN, ${ }^{*}$ Peking University \\ TING YANG, ${ }^{* *}$ Chinese Academy of Sciences
}

\begin{abstract}
In this article we study the parabolic system of equations which is closely related to a multitype branching Brownian motion. Particular attention is paid to the monotone traveling wave solutions of this system. Provided with some moment conditions, we show the existence, uniqueness, and asymptotic behaviors of such waves with speed greater than or equal to a critical value $\underline{c}$ and nonexistence of such waves with speed smaller than $\underline{c}$.
\end{abstract}

Keywords: Multitype branching Brownian motion; spine approach; additive martingale; traveling wave solution

2010 Mathematics Subject Classification: Primary 60J80

Secondary $35 \mathrm{C} 07$

\section{Introduction and main results}

We consider a branching particle system in which there are $d(2 \leq d<+\infty)$ different types of particles. Let $S=\{1,2, \ldots, d\}$ be the set of types. A type $i$ particle splits into offspring particles of all possible types according to a distribution $\left\{p_{k}(i): k \in \mathbb{Z}_{+}^{d}\right\}$ after a lifetime which is exponentially distributed with parameter $a_{i}>0$. All particles engender independent lines of descent. In addition, each particle, when it is alive, diffuses in space $\mathbb{R}$ independently according to a Brownian motion starting from its point of creation. This system is called a multitype branching Brownian motion (MBBM). For more precise configuration of MBBM, see Section 2.

In this article, we assume that each particle reproduces at least one child, which guarantees that the process survives forever with probability one. Suppose that $m_{i j}:=\sum_{k \in \mathbb{Z}_{+}^{d}} p_{k}(i) k_{j}<$ $+\infty$, and that the mean matrix $M=\left(m_{i j}\right)_{i, j \in S}$ is irreducible, i.e. there exists no permutation matrix $S$ such that $S^{-1} M S$ is block triangular. We study the following parabolic system of equations which is strongly related to MBBM:

$$
\frac{\partial u}{\partial t}=\frac{1}{2} \frac{\partial^{2} u}{\partial x^{2}}+\Lambda(\psi(u)-u) .
$$

\footnotetext{
Received 13 September 2012; revision received 21 January 2013.

* Postal address: LMAM School of Mathematical Sciences and Center for Statistical Science, Peking University, Beijing, 100871, P. R. China. Email address: yxren@math.pku.edu.cn

Research supported in part by NNSF of China (Grant numbers 10971003, 11128101, and 11271030) and Specialized Research Fund for the Doctoral Program of Higher Education.

** Postal address: Institute of Applied Mathematics, Academy of Mathematics and Systems Science, Chinese Academy of Sciences, Beijing, 100080, P. R. China. Email address: yangt@amss.ac.cn

Research supported by China Postdoctoral Science Foundation (Grant number 2013M541061).
} 
Here, $u(t, x)=\left(u_{1}(t, x), u_{2}(t, x), \ldots, u_{d}(t, x)\right)^{\top}, \Lambda$ is a diagonal matrix with diagonal entries $\left\{a_{i}: i=1, \ldots, d\right\}$, and $\psi(u)=\left(\psi_{1}(u), \psi_{2}(u), \ldots, \psi_{d}(u)\right)^{\top}$ with

$$
\psi_{i}\left(z_{1}, \ldots, z_{d}\right)=\sum_{k \in \mathbb{Z}_{+}^{d}} p_{k}(i) \prod_{j=1}^{d} z_{j}^{k_{j}}
$$

being the generating function of offspring split by a type $i$ particle. Our primary concern in this article is the solutions satisfying $u(t, x)=w(x-c t)$ where $w$ is a monotone function connecting 0 at $-\infty$ to 1 at $+\infty$. Such solutions are called traveling waves. The analogous object to (1) for a single-type branching Brownian motion is called the Fisher-KolmogorovPetrovski-Piscounov (FKPP) equation. FKPP equation has been studied extensively using both analytic and probabilistic methods (see, for example, [3], [4], [8], [11], and [14]). Among these works, [8] and [11] give proofs for the existence, uniqueness, and asymptotic of traveling wave solutions to the FKPP equation through purely probabilistic arguments. Recently, Kyprianou et al. [12] extended the probabilistic arguments to the traveling wave equations associated to super-Brownian motions with a general branching mechanism.

In this article we outline a probabilistic study on traveling waves of system (1). Our work is strongly guided by the probabilistic arguments in [11] with respect to single-type branching Brownian motions. An important tool of our probabilistic arguments is a representation of the family tree in terms of a suitable size-biased tree with spine. This representation is the continuous-time analogue of the size-biased tree representation introduced by [10]. This continuous-time version is also used in [6] to investigate the evolution of the ancestral types of typical particles for multitype Markov branching processes.

We call $u$ a traveling wave solution with speed $c$ if $u(t, x)$ satisfies (1) and $u$ can be written as $u(t, x)=w(x-c t)=\left(w_{1}(x-c t), \ldots, w_{d}(x-c t)\right)^{\top}$ where $w_{i}(\cdot)$ is a twice continuously differentiable, strictly monotone function increasing from 0 at $-\infty$ to 1 at $+\infty$. For simplicity, $w$ is also called a traveling wave with speed $c$. Obviously, $w$ provides a traveling wave solution to (1) if and only if

$$
\frac{1}{2} \frac{\partial^{2} w}{\partial x^{2}}+c \frac{\partial w}{\partial x}+\Lambda(\psi(w)-w)=0 .
$$

Sometimes, we write $u_{i}(t, x)$ and $w_{i}(x)$ as $u(t, x, i)$ and $w(x, i)$, respectively.

Let $N(t):=\left(N_{1}(t), N_{2}(t), \ldots, N_{d}(t)\right)^{\top}$ be the vector denoting the population sizes of different types at time $t$. Suppose that $m_{i j}(t):=E_{i}\left(N_{j}(t)\right)<+\infty$ for every $i, j \in S$. It is known that the mean matrix $M(t)=\left(m_{i j}(t)\right)_{i, j \in S}$ can be written as

$$
M(t)=\exp (A t)=\sum_{n=0}^{+\infty} \frac{A^{n}}{n !} t^{n}, \quad \text { where } A=\left(a_{i j}\right)_{i, j \in S}, a_{i j}=a_{i}\left(m_{i j}-\delta_{i j}\right) .
$$

It follows from the irreducibility of $M$ that $M(t)$ has positive entries for some $t>0$ (this property is also called 'positive regularity' by [2]). According to the Perron-Frobenius theorem (see [16, Theorem 2.5]), $A$ admits a real eigenvalue $\lambda^{*}>0$ larger than the real part of any other eigenvalue. The so-called Perron's root $\lambda^{*}$ is simple, with a one-dimensional eigenspace, and there are corresponding left and right eigenvectors with positive coordinates. In the following we denote by $\pi$ (respectively $h$ ) the associated left (respectively right) eigenvector with normalization $\langle\pi, h\rangle=\langle\pi, 1\rangle=1$ (here, $\langle\cdot, \cdot\rangle$ denotes the Euclidean inner product).

For $\lambda \neq 0$, define

$$
c_{\lambda}:=\frac{\lambda}{2}+\frac{\lambda^{*}}{\lambda}
$$


which will serve as the speeds of traveling waves. In the following, we deal only with the case $c_{\lambda} \geq 0$. Traveling waves with negative speeds can be analyzed by simple considerations of symmetry. Let $\underline{\lambda}:=\sqrt{2 \lambda^{*}}$. It is easy to see that $c_{\lambda}$ attains a local minimum $\underline{c}=c_{\lambda}=\sqrt{2 \lambda^{*}}$ at $\underline{\lambda}$. We call (2) subcritical, critical, or supercritical according to whether $c$ is less than, equal to, or greater than $\underline{c}$.

Let the configuration of this MBBM at time $t$ be given by the $\mathbb{R} \times S$-valued point process $\left\{\left(X_{v}(t), Y_{v}\right): v \in Z(t)\right\}$, where $Z(t)$ is the set of particles alive at time $t, X_{v}(t)$ is the spatial location of $v$, and $Y_{v}$ is its type. For any $x \in \mathbb{R}$ and $y \in S$, let $P_{x y}$ be the law of the process starting from a single particle of type $y$ at spatial position $x$. Let $E_{x y}$ be the expectation corresponding to $P_{x y}$. To state our main results, we introduce two types of additive martingales which will play an important role in this paper. For any $\lambda \neq 0$, define

$$
W_{\lambda}(t):=\sum_{v \in Z(t)} h_{Y_{v}} \mathrm{e}^{-\lambda\left(X_{v}(t)+c_{\lambda} t\right)} .
$$

From the many-to-one formula (see Proposition 1, below), it is easy to see that $\left\{W_{\lambda}(t), t \geq 0\right\}$ is a positive martingale under $P_{x y}$ and, consequently, the almost sure limit of $W_{\lambda}(t)$ exists. Set $W(\lambda):=\lim _{t \rightarrow+\infty} W_{\lambda}(t)$. Now we define another type of additive martingale:

$$
M_{\lambda}(t):=\sum_{v \in Z(t)} h_{Y_{v}}\left(X_{v}(t)+\lambda t\right) \mathrm{e}^{-\lambda\left(X_{v}(t)+c_{\lambda} t\right)} .
$$

Here, $\left\{M_{\lambda}(t), t \geq 0\right\}$ is a martingale which may take both positive and negative values. We will prove that $M(\lambda):=\lim _{t \rightarrow+\infty} M_{\lambda}(t)$ exists for every $\lambda \geq \underline{\lambda}$ (see Lemma 10, below).

For every $i \in S$, suppose that $\left(\xi_{i 1}, \ldots, \xi_{i d}\right)^{\top}$ is a random vector with the law $\left\{p_{k}(i): k \in\right.$ $\left.\mathbb{Z}_{+}^{d}\right\}$. Now we are ready to state the main results of this paper.

Theorem 1. Suppose that $E\left(\xi_{i j} \log ^{+} \xi_{i j}\right)<+\infty$ for all $i, j \in S$.

(a) When $c>\underline{c}$, there is a unique traveling wave at speed $c$ given by

$$
w(x, y)=E_{x y}[\exp \{-W(\lambda)\}]=E_{0 y}\left[\exp \left\{-\mathrm{e}^{-\lambda x} W(\lambda)\right\}\right], \quad \text { for all }(x, y) \in \mathbb{R} \times S,
$$

where $0<\lambda<\underline{\lambda}$ is the root of the equation $c_{\lambda}=c$. Further, for every $y \in S, 1-w(x, y)$ $\sim h_{y} \mathrm{e}^{-\lambda x}$ as $x \rightarrow+\infty$.

(b) When $c<\underline{c}$, there is no nontrivial traveling wave solution to (1) with speed $c$.

Theorem 2. When $c=\underline{c}$ and $E \xi_{i j}\left(\log ^{+} \xi_{i j}\right)^{2}<+\infty$ for all $i, j \in S$, there is a unique traveling wave at speed $\underline{c}$ given by

$$
\underline{w}(x, y)=E_{x y}[\exp \{-M(\underline{\lambda})\}]=E_{0 y}\left[\exp \left\{-\mathrm{e}^{-\underline{\lambda} x} M(\underline{\lambda})\right\}\right], \quad \text { for all }(x, y) \in \mathbb{R} \times S .
$$

Further, for every $y \in S, 1-\underline{w}(x, y) \sim x h_{y} \mathrm{e}^{-\underline{\lambda} x}$ as $x \rightarrow+\infty$.

Comparing the above theorems with the corresponding results for the FKPP equation (see, for example, [8] and [11]), we see that $\lambda^{*}$ plays the role of $\beta(m-1)$ in the case of a singletype branching Brownian motion, where $\beta$ is the branching rate and $m$ is the mean number of particles split by one particle.

The remainder of this article is structured as follows. In Section 2, we recall the basic setting of family trees and the size-biased trees with spine. We also introduce some known 
results for MBBM, including the so-called many-to-one formula, and McKean representation of traveling wave solutions, which are necessary in the arguments afterwards. In the remaining two sections we concentrate on proofs of Theorem 1 and Theorem 2. To prove that, under some moment conditions, the traveling wave solution can be given in terms of the martingale limit $W(\lambda)$ or $M(\underline{\lambda})$, we first answer when $W(\lambda)$ (in supercritical case) and $M(\underline{\lambda})$ (in critical case) are nondegenerate (see Theorems 3 and 5, respectively).

\section{MBBM and basic facts}

It is known that the family structure of the individuals in a branching process is well expressed by Galton-Watson trees (see, for example, [7]). Each Galton-Watson tree has a single initial ancestor $\varnothing$ and contains all ancestors as well as children of any of its individuals. In order to give other features of our MBBM, we need to introduce the concept of marked Galton-Watson trees. Let $\mathbb{T}$ be the collection of Galton-Watson trees. For each $i \in \mathbb{N}$ where $\mathbb{N}=\{1,2, \ldots\}$, we write $u i$ for the $i$ th child of $u$. We use the notation $v \prec u$ to mean that $v$ is an ancestor of $u$ and $u \in Z(t)$ when $u$ is alive at time $t$. For every $\tau \in \mathbb{T}$, we assume that each particle $u \in \tau$ has a mark $\left(X_{u}, Y_{u}, \sigma_{u}, A_{u}\right)$, where

1. $\sigma_{u}$ is the life time of $u$, which determines the fission time or the death time of particle $u$ as $\zeta_{u}=\sum_{v \prec u} \sigma_{v}+\sigma_{u}\left(\zeta_{\varnothing}=\sigma_{\varnothing}\right)$ and the birth time of $u$ as $b_{u}=\sum_{v \prec u} \sigma_{v}\left(b_{\varnothing}=0\right)$,

2. $Y_{u}$ gives the type of $u$, while $X_{u}:\left[b_{u}, \zeta_{u}\right) \rightarrow \mathbb{R}$ gives the spatial location of $u$ at time $t \in\left[b_{u}, \zeta_{u}\right)$; we also interpret the notation $X_{u}(t)$ as the spatial location of the unique ancestor of $u$ that was alive at time $t \leq \zeta_{u}$,

3. $A_{u}=\left(A_{u}(1), A_{u}(2), \ldots, A_{u}(d)\right)^{\top}$ gives the vector of offspring size split by $u$ when it dies.

We use $(\tau, X, Y, \sigma, A)$ or simply $(\tau, M)$ to denote a marked Galton-Watson tree. Let $\mathcal{T}:=$ $\{(\tau, M): \tau \in \mathbb{T}\}$. Define

$$
\begin{gathered}
\mathcal{F}_{t}:=\sigma\left\{\left[u, Y_{u}, \sigma_{u}, A_{u},\left(X_{u}(s), s \in\left[b_{u}, \zeta_{u}\right)\right): u \in \tau \in \mathbb{T} \text { with } \zeta_{u} \leq t\right]\right. \text { and } \\
\left.\left[u, Y_{u},\left(X_{u}(s), s \in\left[b_{u}, t\right)\right): u \in \tau \in \mathbb{T} \text { with } t \in\left[b_{u}, \zeta_{u}\right)\right]\right\} .
\end{gathered}
$$

Set $\mathcal{F}=\bigcup_{t \geq 0} \mathcal{F}_{t}$. There is a unique probability measure $P$ on $(\mathcal{T}, \mathcal{F})$ such that the system is initiated by a single ancestor and evolves as a MBBM defined in Section 1.

Now we extend the probability space $(\mathcal{T}, \widetilde{F}, P)$ to $(\widetilde{\mathcal{T}}, \widetilde{\mathcal{F}}, \widetilde{P})$ defined below. For any $\tau \in \mathbb{T}$, we can select an infinite line of descent $\varepsilon=\left\{\varepsilon_{0}=\varnothing, \varepsilon_{1}, \varepsilon_{2}, \ldots\right\}$, where $\varepsilon_{n+1} \in \tau$ is a child of $\varepsilon_{n} \in \tau$ for $n \in\{0,1,2, \ldots\}$. Such a genealogical line is called a spine. We write $u \in \varepsilon$ to mean that $u=\varepsilon_{k}$ for some $k \in \mathbb{Z}_{+}$. We use $\widetilde{\mathcal{T}}=\{(\tau, M, \varepsilon): \varepsilon \subset \tau \in \mathbb{T}\}$ to denote the set of all marked trees with distinguished spines.

We use $\widetilde{Y}=\left(\widetilde{Y}_{t}, t \geq 0\right)$ to denote the type process of the spine, $\widetilde{X}=\left(\widetilde{X}_{t}, t \geq 0\right)$ to denote the spatial movement of the spine, and $n=\left(n_{t}, t \geq 0\right)$ to denote the counting process of fission times along the spine. Let $\operatorname{node}_{t}(\varepsilon):=u$ if $u \in \varepsilon$ is the node in the spine that is alive at time $t$. Note that, for $u \in \varepsilon, Y_{u}=\widetilde{Y}_{b_{u}}=\widetilde{Y}_{\zeta_{u}-}$.

If $u \in \varepsilon$, then at the fission time $\zeta_{u}$, it gives birth to $\left\langle A_{u}, 1\right\rangle$ offspring, one of which continuing the spine (we write this node simply as $u+1$ ) while the others going on to create independent subtrees. Let $O_{u}$ be the set of $u$ 's children except the one in the spine. For any $j \in\left\{1,2, \ldots,\left\langle A_{u}, 1\right\rangle\right\}$ such that $u j \in O_{u}$, we use $(\tau, M)_{j}^{u}$ to denote the marked tree rooted at $u j$. 
Now we introduce some filtrations on $\widetilde{\mathcal{T}}$ that we shall use later. First note that $\left\{\mathcal{F}_{t}, t \geq 0\right\}$ is also a filtration on $\widetilde{\mathcal{T}}$. Define

$$
\begin{gathered}
\widetilde{\mathcal{F}}_{t}:=\sigma\left\{\mathcal{F}_{t},\left(\operatorname{node}_{s}(\varepsilon), s \leq t\right)\right\}, \\
g_{t}:=\sigma\left\{\widetilde{Y}_{s}, \widetilde{X}_{s}: 0 \leq s \leq t\right\}, \quad g_{t}:=\sigma\left\{\widetilde{Y}_{s}: 0 \leq s \leq t\right\}, \quad g_{t} \tilde{X}:=\sigma\left\{\widetilde{X}_{s}: 0 \leq s \leq t\right\}, \\
\widehat{g}_{t}:=\sigma\left\{g_{t},\left(\operatorname{node}_{s}(\varepsilon), s \leq t\right),\left(\zeta_{u}, u \prec \operatorname{node}_{t}(\varepsilon)\right)\right\}, \\
\widetilde{g}_{t}:=\sigma\left\{\widehat{g}_{t},\left(A_{u}, u \prec \operatorname{node}_{t}(\varepsilon)\right)\right\} .
\end{gathered}
$$

Set $\widetilde{\mathcal{F}}=\bigcup_{t \geq 0} \widetilde{\mathcal{F}}_{t}, g=\bigcup_{t \geq 0} g_{t}, \widehat{g}=\bigcup_{t \geq 0} \widehat{g}_{t}$, and $\widetilde{g}=\bigcup_{t \geq 0} \widetilde{g}_{t}$.

Now we shall extend the probability measure $P$ on $(\mathcal{T}, \mathcal{F})$ to a probability measure $\widetilde{P}$ on $(\widetilde{\mathcal{T}}, \widetilde{\mathcal{F}})$ such that the spine is a single genealogical line of descent chosen from the underlying tree. Enlightened by [13], when a spine node $u$ of type $i$ dies, we pick one of its children at random to be the successor on the spine. Specifically, children are picked with probabilities proportional to $h_{j}$ when their type is $j$. This means, when $u \in \tau$, we have

$$
\operatorname{Prob}\left(u \in \varepsilon \mid \mathcal{F}_{t}\right)=\prod_{v \prec u} \frac{h_{Y_{v+1}}}{\left\langle A_{v}, h\right\rangle} .
$$

To define $\widetilde{P}$ we recall the following representation from [13].

Lemma 1. Every $\widetilde{\mathcal{F}}_{t}$-measurable function $f$ can be written as

$$
f=\sum_{u \in Z(t)} f_{u} \mathbf{1}_{\{u \in \varepsilon\}}
$$

where $f_{u}$ is $\mathcal{F}_{t}$-measurable.

Definition 1. We define the probability measure $\widetilde{P}$ on $(\widetilde{\mathcal{T}}, \widetilde{\mathcal{F}})$ by

$$
\int_{\widetilde{\mathcal{T}}} f \mathrm{~d} \widetilde{P}=\int_{\widetilde{\mathcal{T}}} \sum_{u \in Z(t)} f_{u} \prod_{v \prec u} \frac{h_{Y_{v+1}}}{\left\langle A_{v}, h\right\rangle} \mathrm{d} P,
$$

for each $f \in \widetilde{\mathcal{F}}_{t}$ with representation (4).

Intuitively, following the above method of choosing spine nodes, the type process of the spine $\widetilde{Y}$ is a continuous-time Markov process valued in $S$, which stays at any state $i \in S$ for an exponential time with parameter $a_{i}$, and then transits to state $j$ with probability $P(i, j):=$ $\sum_{k \in \mathbb{Z}_{+}^{d}} p_{k}(i) k_{j} h_{j} /\langle k, h\rangle$. Given $\widehat{g_{t}}$, the trajectory of $\widetilde{Y}$, the node of the spine and the birth time of each spine node before time $t$ are determined. Then we have

$$
\widetilde{P}\left(A_{v}=k_{v} \text {, for all } v \prec \varepsilon_{n_{t}} \mid \widehat{g}_{t}\right)=\prod_{v \prec \varepsilon_{n_{t}}} \frac{p_{k_{v}}\left(Y_{v}\right)}{P\left(Y_{v}, Y_{v+1}\right)} \frac{k_{v}\left(Y_{v+1}\right) h_{Y_{v+1}}}{\left\langle k_{v}, h\right\rangle},
$$

where $k_{v}=\left(k_{v}(1), k_{v}(2), \ldots, k_{v}(d)\right)^{\top} \in \mathbb{Z}_{+}^{d}$. Now we construct a probability measure $\widetilde{P}$ on $\widetilde{\mathcal{F}_{t}}$ by

$$
\begin{aligned}
\left.\mathrm{d} \widetilde{P}(\tau, M, \varepsilon)\right|_{\widetilde{F}_{t}}= & \mathrm{d} \mathbb{P}(\tilde{Y}) \mathrm{d} \mathbb{B}(\tilde{X}) \prod_{v \prec \varepsilon_{n_{t}}} \frac{p_{A_{v}}\left(Y_{v}\right)}{P\left(Y_{v}, Y_{v+1}\right)} \frac{A_{v}\left(Y_{v+1}\right) h_{Y_{v+1}}}{\left\langle A_{v}, h\right\rangle} \\
& \times \prod_{v \prec \varepsilon_{n_{t}}}\left[\frac{1}{A_{v}\left(Y_{v+1}\right)} \prod_{j: v j \in O_{v}} \mathrm{~d} P_{\widetilde{X}_{\zeta v} Y_{v j}}^{t-\zeta_{v}}\left((\tau, M)_{j}^{v}\right)\right] .
\end{aligned}
$$


Here, $\mathbb{B}(\tilde{X})$ is the law of a standard Brownian motion and $\mathbb{P}(\tilde{Y})$ is the law of the type process $\tilde{Y}$. The decomposition of $\widetilde{P}$ suggests the following intuitive description of the system under the measure $\widetilde{P}$.

1. The spine's type process $\widetilde{Y}$ moves as a continuous-time Markov process taking values in $S$ according to the measure $\mathbb{P}$. The generator $G=\left(g_{i j}\right)_{i, j \in S}$ of $\widetilde{Y}$ is given by $g_{i j}=$ $a_{i}\left(P(i, j)-\delta_{i j}\right)$. The spine's spatial movement $\widetilde{X}$ is a standard Brownian motion.

2. The fission time $\zeta_{v}$ of node $v$ in the spine is exactly the jumping time of the spine's type process $\widetilde{Y}$, i.e. the life time $\sigma_{v}$ of $v$ is exponentially distributed with parameter $a_{\widetilde{Y}_{b_{v}}}$. (Here, $\widetilde{Y}$ may jump from $i$ to itself at jumping time according to generator $G$.)

3. At the fission time of node $v$ in the spine, the single spine particle is replaced by a random vector $A_{v}$ of offspring with $A_{v}$ being distributed according to the law $\left(p_{k}\left(\widetilde{Y}_{\zeta_{v}-}\right)\right)_{k \in \mathbb{Z}_{+}^{d}}$, and a type $j$ child is picked to be the next spine node with probability $h_{j} /\left\langle A_{v}, h\right\rangle$.

4. The remaining $\left\langle A_{v}, 1\right\rangle-1$ nonspine children of $v$ give rise to independent subtrees $(\tau, M)_{j}^{v}$ for $v j \in O_{v}$, each evolving as an independent subtree determined by the probability $P_{\widetilde{X}_{\zeta v} Y_{v j}}$ shifted to the time of creation.

Note that $\{N(t), t \geq 0\}$ is a multitype branching process, where $N(t)$ denotes the population size vector at time $t$. We have the following lemma.

Lemma 2. (Athreya [1, Proposition 2].) The martingale

$$
\left\{w(t):=\frac{\mathrm{e}^{-\lambda^{*} t}\langle N(t), h\rangle}{\langle N(0), h\rangle}: t \geq 0\right\}
$$

is a nonnegative martingale with respect to $\left\{\mathcal{F}_{t}: t \geq 0\right\}$.

In order to make the principles of the measure change method clear, we introduce a technical lemma which follows from an elementary argument.

Lemma 3. Suppose that $\tilde{\mu}_{1}$ and $\tilde{\mu}_{2}$ are two probability measures defined on the same space $(\Omega, \widetilde{\mathcal{F}})$ with Radon-Nikodym derivative $g$ such that $\mathrm{d} \widetilde{\mu}_{2}=g \mathrm{~d} \widetilde{\mu}_{1}$. If $\mathcal{F}$ is a sub- $\sigma$-field of $\widetilde{\mathcal{F}}$, then the two measures $\mu_{1}:=\left.\tilde{\mu}_{1}\right|_{\mathcal{F}}$ and $\mu_{2}:=\left.\tilde{\mu}_{2}\right|_{\mathcal{F}}$ on $(\Omega, \mathcal{F})$ are related by the conditional expectation operation $\mathrm{d} \mu_{2}=\widetilde{\mu}_{1}(g \mid \mathcal{F}) \mathrm{d} \mu_{1}$.

Noting that $w(t)$ is a nonnegative mean-one martingale, we can define a probability measure $Q$ on $(\mathcal{T}, \mathcal{F})$ by

$$
\left.\mathrm{d} Q\right|_{\mathscr{F}_{t}}=\left.w(t) \mathrm{d} P\right|_{\mathcal{F}_{t}} .
$$

Lemma $\underset{\widetilde{F}}{3}$ implies that, if we want to extend $Q$ defined by (6) to a probability measure $\widetilde{Q}$ on $(\widetilde{\widetilde{T}}, \widetilde{\mathcal{F}})$, we need to construct a nonnegative martingale $\widetilde{w}(t)$ with respect to $\left\{\widetilde{\mathcal{F}}_{t}: t \geq 0\right\}$ satisfying

$$
\left.\mathrm{d} \widetilde{Q}\right|_{\widetilde{\mathcal{F}}_{t}}=\left.\widetilde{w}(t) \mathrm{d} \widetilde{P}\right|_{\widetilde{\mathcal{F}}_{t}}
$$

and

$$
\widetilde{P}\left(\widetilde{w}(t) \mid \mathcal{F}_{t}\right)=w(t) .
$$

According to Lemma 1, $\widetilde{w}(t)$ can be written as $\widetilde{w}(t)=\sum_{v \in Z(t)} w_{v} \mathbf{1}_{\{v \in \varepsilon\}}$, where $w_{v}$ is $\mathcal{F}_{t}$-measurable. Immediately we have $\widetilde{P}\left(\widetilde{w}(t) \mid \mathcal{F}_{t}\right)=\sum_{v \in Z(t)} w_{v} \prod_{u \prec v} h_{Y_{u+1}} /\left\langle A_{u}, h\right\rangle$ by 
Definition 1. Since $w(t)=\sum_{v \in Z(t)} \mathrm{e}^{-\lambda^{*} t} h_{Y_{v}} /\langle N(0), h\rangle$, (8) implies that

$$
w_{v}=\frac{h_{Y_{v}}}{\mathrm{e}^{\lambda^{*} t}\langle N(0), h\rangle}\left(\prod_{u \prec v} \frac{h_{Y_{u+1}}}{\left\langle A_{u}, h\right\rangle}\right)^{-1}=\mathrm{e}^{-\lambda^{*} t} \prod_{u \prec v} \frac{\left\langle A_{u}, h\right\rangle}{h_{Y_{u}}},
$$

and, consequently,

$$
\widetilde{w}(t)=\mathrm{e}^{-\lambda^{*} t} \prod_{v \prec \varepsilon_{n_{t}}} \frac{\left\langle A_{v}, h\right\rangle}{h_{\widetilde{Y}_{\zeta v}}} .
$$

Next we will prove that $\{\widetilde{w}(t): t \geq 0\}$ is indeed a martingale with respect to $\left\{\widetilde{\mathcal{F}}_{t}: t \geq 0\right\}$. First of all, for each type $i \in S$, we introduce the size-biased distribution

$$
\hat{p}_{k}(i):=\frac{p_{k}(i)\langle k, h\rangle}{\left(1+\lambda^{*} / a_{i}\right) h_{i}} .
$$

It is a probability distribution since $\sum_{k \in \mathbb{Z}_{+}^{d}} p_{k}(i)\langle k, h\rangle=\sum_{j=1}^{d} m_{i j} h_{j}=\left(1+\lambda^{*} / a_{i}\right) h_{i}$ for every $i \in S$ (the last equality follows from the fact that $h$ is the right eigenvector of $A$ with respect to $\left.\lambda^{*}\right)$. For any $i, j \in S$, define

$$
\widehat{P}(i, j):=\sum_{k \in \mathbb{Z}_{+}^{d}} \hat{p}_{k}(i) \frac{k_{j} h_{j}}{\langle k, h\rangle}=\frac{m_{i j} h_{j}}{\left(1+\lambda^{*} / a_{i}\right) h_{i}} .
$$

It is easy to see that $\{\widehat{P}(i, j): i, j \in S\}$ is a family of transition probabilities.

Lemma 4. Suppose that $(\tilde{Y}, \mathbb{P})$ is defined as before. Define

$$
m_{t}:=\mathrm{e}^{-\lambda^{*} t} \prod_{v \prec \varepsilon_{n_{t}}}\left(1+\frac{\lambda^{*}}{a_{Y_{v}}}\right) \frac{\widehat{P}\left(Y_{v}, Y_{v+1}\right)}{P\left(Y_{v}, Y_{v+1}\right)}, \quad t \geq 0 .
$$

Then $\left\{m_{t}, t \geq 0\right\}$ is a nonnegative mean-one martingale with respect to $\left\{\widehat{g}_{t}, t \geq 0\right\}$. We define another probability measure $\hat{\mathbb{P}}$ by

$$
\left.\mathrm{d} \hat{\mathbb{P}}\right|_{\widehat{g}_{t}}=\left.m_{t} \mathrm{~d} \mathbb{P}\right|_{\widehat{g}_{t}} .
$$

Then, under $\hat{\mathbb{P}}, \tilde{Y}$ moves as a continuous-time Markov process with generator $\hat{g}_{i j}:=\left(a_{i}+\right.$ $\left.\lambda^{*}\right)\left(\widehat{P}(i, j)-\delta_{i j}\right)$ for $i, j \in S$.

Proof. Suppose that $f: S \rightarrow \mathbb{R}$ is a bounded measurable function. For every $i \in S$, define $u(t, i):=\mathbb{E}^{i}\left[f\left(\widetilde{Y}_{t}\right) m_{t}\right]$ where $\mathbb{P}^{i}(\cdot):=\mathbb{P}\left(\cdot \mid \widetilde{Y}_{0}=i\right)$ with associated expectation operator $\mathbb{E}^{i}$. We use $\tau$ to denote the first jumping time of $\widetilde{Y}$. Then, by the strong Markov property, $u(t, i)$ can be written as

$$
\begin{aligned}
u(t, i) & =\mathbb{E}^{i}\left[f\left(\tilde{Y}_{t}\right) m_{t} \mathbf{1}_{\{t<\tau\}}\right]+\mathbb{E}^{i}\left[f\left(\tilde{Y}_{t}\right) m_{t} \mathbf{1}_{\{t \geq \tau\}}\right] \\
& =f(i) \mathrm{e}^{-\left(a_{i}+\lambda^{*}\right) t}+\int_{0}^{t} \mathrm{e}^{-\left(a_{i}+\lambda^{*}\right) s}\left(a_{i}+\lambda^{*}\right) \sum_{j \in S} \widehat{P}(i, j) u(t-s, j) \mathrm{d} s \\
& =f(i) \mathrm{e}^{-\left(a_{i}+\lambda^{*}\right) t}+\int_{0}^{t} \mathrm{e}^{-\left(a_{i}+\lambda^{*}\right)(t-s)}\left(a_{i}+\lambda^{*}\right) \sum_{j \in S} \widehat{P}(i, j) u(s, j) \mathrm{d} s .
\end{aligned}
$$


Therefore, $u(t, i)$ satisfies

$$
\frac{\partial u}{\partial t}=\left(a_{i}+\lambda^{*}\right) \sum_{j \in S}\left(\widehat{P}(i, j)-\delta_{i j}\right) u(t, j),
$$

with $u(0, i)=f(i)$. In particular, if we pick $f \equiv 1$, from the uniqueness of the bounded solution to (11), we obtain that $\mathbb{E}^{i} m_{t} \equiv 1$, which together with the Markov property of $\tilde{Y}$ under $\mathbb{P}$ implies that $m_{t}$ is a martingale. Thus, the measure $\hat{\mathbb{P}}$ is well defined. From (11) we see that, under $\hat{\mathbb{P}}, \widetilde{Y}$ is a Markov process with generator $\hat{g}_{i j}$. In other words, under probability measure $\hat{\mathbb{P}}$, $\widetilde{Y}$ can be interpreted as a Markov process which stays at each state $i \in S$ for an exponential time with parameter $a_{i}+\lambda^{*}$, and then transits to state $j$ with probability $\widehat{P}(i, j)$. This completes the proof.

Just as we did before, we can construct a probability measure $\widetilde{Q}$ on $(\widetilde{\mathcal{T}}, \widetilde{\mathcal{F}})$ by

$$
\begin{aligned}
\left.\mathrm{d} \widetilde{Q}(\tau, M, \varepsilon)\right|_{\widetilde{F}_{t}=} & \mathrm{d} \hat{\mathbb{P}}(\tilde{Y}) \mathrm{d} \mathbb{B}(\tilde{X}) \prod_{v \prec \varepsilon_{n_{t}}} \frac{\hat{p}_{A_{v}}\left(Y_{v}\right)}{\widehat{P}\left(Y_{v}, Y_{v+1}\right)} \frac{A_{v}\left(Y_{v+1}\right) h_{Y_{v+1}}}{\left\langle A_{v}, h\right\rangle} \\
& \times \prod_{v \prec \varepsilon_{n_{t}}}\left[\frac{1}{A_{v}\left(Y_{v+1}\right)} \prod_{j: v j \in O_{v}} \mathrm{~d} P_{\widetilde{X}_{\zeta v} Y_{v j}}^{t-\zeta_{v}}\left((\tau, M)_{j}^{v}\right)\right],
\end{aligned}
$$

under which the system can be described as follows.

1. The spine's type process $\tilde{Y}$ moves as a continuous-time Markov process valued on $S$ according to the measure $\hat{\mathbb{P}}$. The generator of $\widetilde{Y}$ is given by $\hat{g}_{i j}=\left(a_{i}+\lambda^{*}\right)\left(\widehat{P}(i, j)-\delta_{i j}\right)$. The spine's spatial movement $\widetilde{X}$ is a standard Brownian motion.

2. The fission time $\zeta_{v}$ of node $v$ in the spine is exactly the jumping time of the spine's type process $\widetilde{Y}$, i.e. $\sigma_{v}$ has an exponential distribution with parameter $a_{\widetilde{Y}_{b_{v}}}+\lambda^{*}$.

3. At the fission time of node $v$ in the spine, the single spine particle is replaced by a random vector $A_{v}$ of offspring with $A_{v}$ being distributed according to the law $\left(\hat{p}_{k}\left(\widetilde{Y}_{\zeta_{v}-}\right)\right)_{k \in \mathbb{Z}_{+}^{d}}$, and a type $j$ particle from the offspring of $v$ will be picked to be the next spine node with probability $h_{j} /\left\langle A_{v}, h\right\rangle$.

4. The remaining $\left\langle A_{v}, 1\right\rangle-1$ nonspine children of $v$ give rise to independent subtrees $(\tau, M)_{j}^{v}$ for $v j \in O_{v}$, each evolving as an independent subtree determined by the probability $P_{\widetilde{X}_{\zeta v} Y_{v j}}$ shifted to the time of creation.

Applying (9), (10), and (5) to (12), we can easily get (7). Therefore, $\{\widetilde{w}(t): t \geq 0\}$ is a nonnegative martingale with respect to $\left\{\widetilde{\mathcal{F}}_{t}: t \geq 0\right\}$. The following result is a byproduct of the above spine construction. The proof is much the same as [6, Theorem 4.1] in the case of multitype Markov branching processes. We omit the details here.

Proposition 1. (Many-to-one formula for MBBM.) For any measurable function $f: \mathbb{R} \times S \rightarrow$ $\mathbb{R}$, we have

$$
E_{x y}\left(\sum_{u \in Z(t)} f\left(X_{u}(t), Y_{u}\right)\right)=\widehat{E}_{x y}\left(f\left(\widetilde{X}_{t}, \widetilde{Y}_{t}\right) \frac{h_{\widetilde{Y}_{0}}}{h_{\widetilde{Y}_{t}}} \mathrm{e}^{\lambda^{*} t}\right)
$$


Here, $\widehat{E}_{x y}$ denotes the law of one particle motion where the type process $\widetilde{Y}$ moves as a Markov process starting from $y$ with generator

$$
\hat{g}_{i j}:=\left(a_{i}+\lambda^{*}\right)\left(\widehat{P}(i, j)-\delta_{i j}\right),
$$

for $i, j \in S$, while the spatial location process $\widetilde{X}$ moves as a Brownian motion starting from $x$ and is independent of $\widetilde{Y}$.

Lemma 5. (McKean representation.) If $u(t, x, y) \in[0,1]$ is twice continuously differentiable in $x$ and satisfies the parabolic system of equations (1) with initial condition $u(0, x, y)=$ $f(x, y)$, then $u$ has a McKean representation

$$
u(t, x, y)=E_{x y}\left(\prod_{u \in Z(t)} f\left(X_{u}(t), Y_{u}\right)\right) .
$$

The proof is similar to that of [3, Theorem 1.36]. We omit the details here.

Lemma 6. Suppose that $c \in \mathbb{R}$ and $w(x, y)$ is a bounded function satisfying $0 \leq w(x, y) \leq 1$ for any $(x, y) \in \mathbb{R} \times S$. Let $u(t, x, y):=w(x-c t, y)$. Then $u$ satisfies (1) if and only if

$$
w(x, y)=E_{x y}\left[\prod_{u \in Z(t)} w\left(X_{u}(t)+c t, Y_{u}\right)\right] .
$$

Proof. By Lemma 5, we only need to show the sufficiency. Let $T_{t}$ denote the semi-group of one-dimensional Brownian motion and $\tau$ the split time of the root. We have

$$
\begin{aligned}
u(t, x, y) & =E_{(x-c t)}\left(\prod_{u \in Z(t)} w\left(X_{u}(t)+c t, Y_{u}\right)\right) \\
& =E_{x y}\left(\prod_{u \in Z(t)} w\left(X_{u}(t), Y_{u}\right)\right) \\
& =E_{x y}\left(\prod_{u \in Z(t)} w\left(X_{u}(t), Y_{u}\right) \mathbf{1}_{\{\tau \leq t\}}\right)+E_{x y}\left(\prod_{u \in Z(t)} w\left(X_{u}(t), Y_{u}\right) \mathbf{1}_{\{\tau>t\}}\right) \\
& =\int_{0}^{t} a_{y} \mathrm{e}^{-a_{y} s} T_{s} \psi_{y}\left(u_{t-s}\right)(x) \mathrm{d} s+\mathrm{e}^{-a_{y} t} T_{t} w_{y}(x)
\end{aligned}
$$

where, for each $s \geq 0, u_{s}$ is a function from $\mathbb{R}$ to $\mathbb{R}^{d}$ defined by $u_{s}(x):=u(s, x)=$ $(u(s, x, 1), \ldots, u(s, x, d))^{\top}$. Therefore, $u(t, x, y)$ solves (1).

\section{Proof of Theorem 1}

Recall that, for any $\lambda \neq 0$,

$$
W_{\lambda}(t):=\sum_{u \in Z(t)} h_{Y_{u}} \mathrm{e}^{-\lambda\left(X_{u}(t)+c_{\lambda} t\right)} .
$$

It follows from Proposition 1 that $\left\{W_{\lambda}(t), t \geq 0\right\}$ is a positive martingale and thus has an almost sure limit denoted by $W(\lambda)$. The following theorem answers when $W(\lambda)$ is nondegenerate, 
which will be used to give explicit expressions of traveling wave solutions in the supercritical case.

Theorem 3. (a) If $|\lambda| \geq \underline{\lambda}$, then $W(\lambda)=0 P_{x y}$-almost surely.

(b) Suppose that $0<|\lambda|<\underline{\lambda}$. If $E\left(\xi_{i j} \log ^{+} \xi_{i j}\right)<+\infty$ for all $i, j \in S$, then $W_{\lambda}(t)$ converges to $W(\lambda)$ in $L^{1}\left(P_{x y}\right)$, and $P_{x y}(W(\lambda)=0)=0$. Otherwise, if $E\left(\xi_{i j} \log ^{+} \xi_{i j}\right)=+\infty$ for some $i, j \in S$, then $W(\lambda)=0 P_{x y}$-almost surely.

Remark 1. It suffices to prove the claims for $P_{0 y}$. In this article, we only deal with the case $\lambda>0$. The case $\lambda<0$ can be analyzed by simple considerations of symmetry.

For any $\lambda>0$, through similar techniques as used in Section 2 , we can construct a probability measure $\widetilde{Q}_{0 y}^{\lambda}$ on $(\widetilde{\mathcal{T}}, \widetilde{\mathcal{F}})$ such that

$$
\left.\mathrm{d} Q_{0 y}^{\lambda}\right|_{\mathcal{F}_{t}}=\left.\frac{W_{\lambda}(t)}{W_{\lambda}(0)} \mathrm{d} P_{0 y}\right|_{\mathcal{F}_{t}},
$$

where $Q_{0 y}^{\lambda}:=\widetilde{Q}_{0 y}^{\lambda} \mid \mathcal{F}$. In fact, $\widetilde{Q}_{0 y}^{\lambda}$ has the following decomposition:

$$
\begin{aligned}
\left.\mathrm{d} \widetilde{Q}_{0 y}^{\lambda}(\tau, M, \varepsilon)\right|_{\widetilde{\mathcal{F}}_{t}}= & \mathrm{d} \hat{\mathbb{P}}_{y}(\tilde{Y}) \mathrm{d} \mathbb{B}^{-\lambda}(\tilde{X}) \prod_{v \prec \varepsilon_{n_{t}}} \frac{\hat{p}_{A_{v}}\left(Y_{v}\right)}{\widehat{P}\left(Y_{v}, Y_{v+1}\right)} \frac{A_{v}\left(Y_{v+1}\right) h_{Y_{v+1}}}{\left\langle A_{v}, h\right\rangle} \\
& \times \prod_{v \prec \varepsilon_{n_{t}}}\left[\frac{1}{A_{v}\left(Y_{v+1}\right)} \prod_{j: v j \in O_{v}} \mathrm{~d} P_{\widetilde{X}_{\zeta v} \widetilde{\zeta}_{v j}}^{t-\zeta_{v}}\left((\tau, M)_{j}^{v}\right)\right] .
\end{aligned}
$$

Here, $\left(\widetilde{X}, \mathbb{B}^{-\lambda}\right)$ is a standard Brownian motion with drift $-\lambda$, and $\left(\widetilde{Y}, \hat{\mathbb{P}}_{y}\right)$ is a continuous-time Markov chain starting from $y$ with generator $\hat{g}_{i j}=\left(a_{i}+\lambda^{*}\right)\left(\widehat{P}(i, j)-\delta_{i j}\right)$. For each $v j \in O_{v}$, $(\tau, M)_{j}^{v}$ evolves as an independent subtree determined by the probability $P_{\widetilde{X}_{\zeta v}} Y_{v j}$ shifted to the time of creation.

Lemma 7. We have the following spine decomposition for the martingale $W_{\lambda}(t)$ :

$$
\widetilde{Q}_{0 y}^{\lambda}\left(W_{\lambda}(t) \mid \tilde{g}\right)=h_{\widetilde{Y}_{t}} \mathrm{e}^{-\lambda\left(\tilde{X}(t)+c_{\lambda} t\right)}+\sum_{j \in S} \sum_{v \prec \varepsilon_{n_{t}}}\left(A_{v}(j)-\delta_{Y_{v+1} j}\right) h_{j} \mathrm{e}^{-\lambda\left(\widetilde{X}\left(\zeta_{v}\right)+c_{\lambda} \zeta_{v}\right)}
$$

Proof. Here, $W_{\lambda}(t)$ can be written as

$$
\begin{aligned}
W_{\lambda}(t) & =h_{\widetilde{Y}_{t}} \mathrm{e}^{-\lambda\left(\widetilde{X}(t)+c_{\lambda} t\right)}+\sum_{u \in Z(t), u \notin \varepsilon} h_{Y_{u}} \mathrm{e}^{-\lambda\left(X_{u}(t)+c_{\lambda} t\right)} \\
& =h_{\widetilde{Y}_{t}} \mathrm{e}^{-\lambda\left(\widetilde{X}(t)+c_{\lambda} t\right)}+\sum_{v \prec \varepsilon_{n_{t}}} \sum_{j: v j \in O_{v}} \sum_{u \in Z(t), u \in(\tau, M)_{j}^{v}} h_{Y_{u}} \mathrm{e}^{-\lambda\left(X_{u}(t)+c_{\lambda} t\right)} .
\end{aligned}
$$

The first equality is clearly true since one of the particles $u \in Z(t)$ must stay in the spine. The second follows from partitioning the particles into distinct subtrees that were born by the spine nodes before time $t$. Recall that $\widetilde{g}$ contains all information about the spine nodes; by taking 
the $\widetilde{Q}_{0 y}^{\lambda}$ conditional expectation of $W_{\lambda}(t)$, we have

$$
\begin{aligned}
& \widetilde{Q}_{0 y}^{\lambda}\left(W_{\lambda}(t) \mid \widetilde{g}\right) \\
&=h_{\widetilde{Y}_{t}} \mathrm{e}^{-\lambda\left(\tilde{X}(t)+c_{\lambda} t\right)}+\widetilde{Q}_{0 y}^{\lambda}\left(\sum_{v \prec \varepsilon_{n_{t}}} \sum_{j: v j \in O_{v}} \sum_{u \in Z(t), u \in(\tau, M)_{j}^{v}} h_{Y_{u}} \mathrm{e}^{\left.-\lambda\left(X_{u}(t)+c_{\lambda} t\right) \mid \widetilde{g}\right)}\right. \\
&=h_{\widetilde{Y}_{t}} \mathrm{e}^{-\lambda\left(\tilde{X}(t)+c_{\lambda} t\right)}+\sum_{v \prec \varepsilon_{n t}} \sum_{j: v j \in O_{v}} h_{Y_{v j}} \mathrm{e}^{-\lambda\left(\tilde{X}\left(\zeta_{v}\right)+c_{\lambda} \zeta_{v}\right)} \\
& \quad \times \widetilde{Q}_{0 y}^{\lambda}\left(\sum_{u \in Z(t), u \in(\tau, M)_{j}^{v}} \frac{h_{Y_{u}}}{h_{Y_{v j}}} \mathrm{e}^{-\lambda\left(X_{u}(t)-\tilde{X}\left(\zeta_{v}\right)+c_{\lambda}\left(t-\zeta_{v}\right)\right)} \mid \tilde{g}\right) .
\end{aligned}
$$

From the decomposition of d $\widetilde{Q}_{0 y}^{\lambda}$, we observe that, under $\widetilde{Q}_{0 y}^{\lambda}$, the subtrees coming off the spine evolves as if under the measure $P_{0 y}$. Therefore,

$$
\widetilde{Q}_{0 y}^{\lambda}\left(\sum_{u \in Z(t), u \in(\tau, M)_{j}^{v}} \frac{h_{Y_{u}}}{h_{Y_{v j}}} \mathrm{e}^{-\lambda\left(X_{u}(t)-\widetilde{X}\left(\zeta_{v}\right)+c_{\lambda}\left(t-\zeta_{v}\right)\right)} \mid \tilde{g}\right)=1
$$

This equality is true because the additive expression being evaluated on the subtrees is just a shifted form of the martingale $W_{\lambda}(t)$. We complete the proof.

Lemma 8. (Durret [5, p. 241].) Suppose that $\mu$ and $v$ are two probability measures on a measurable space $(\Omega, \mathcal{F})$ with filtration $\left(\mathcal{F}_{t}\right)_{t \geq 0}$, such that $\left.\mathrm{d} \mu\right|_{\mathcal{F}_{t}}=\left.M(t) \mathrm{d} \nu\right|_{\mathcal{F}_{t}}$ for all $t \geq 0$. Let $M_{\infty}:=\limsup _{t \rightarrow+\infty} M(t)$. Then $\nu\left(M_{\infty}=0\right)=1$ if and only if $\mu\left(M_{\infty}=+\infty\right)=1$, and $\int_{\Omega} M_{\infty} \mathrm{d} \nu=1$ if and only if $\mu\left(M_{\infty}<+\infty\right)=1$.

Proof of Theorem 3. (a) If $\lambda \geq \underline{\lambda}>0$, then $\lambda \geq c_{\lambda}$. Obviously we have

$$
W_{\lambda}(t) \geq h_{\widetilde{Y}_{t}} \mathrm{e}^{-\lambda \tilde{X}(t)-\left(\lambda^{2} / 2+\lambda^{*}\right) t} \geq C_{0} \mathrm{e}^{-\lambda t\left(\tilde{X}(t) / t+c_{\lambda}\right)},
$$

for some constant $C_{0}>0$. Note that $\lim _{t \rightarrow+\infty} \tilde{X}(t) / t=-\lambda$ and $\liminf _{t \rightarrow+\infty} \tilde{X}(t)+$ $\lambda t=-\infty$, since $\widetilde{X}$ moves as a Brownian motion with drift $-\lambda$ under $\widetilde{Q}_{0 y}^{\lambda}$. Thus, we have $\widetilde{Q}_{0 y}^{\lambda}\left(\lim \sup _{t \rightarrow+\infty} W_{\lambda}(t)=+\infty\right)=1$. In view of Lemma 8 , we have $P_{0 y}(W(\lambda)=0)=1$.

(b) If $0<\lambda<\underline{\lambda}$, then $\lambda<c_{\lambda}$. Suppose that $E\left(\xi_{i j} \log ^{+} \xi_{i j}\right)=+\infty$ for some $i, j \in$ $S$. First note that at each fission time of the spine, we have the lower bound $W_{\lambda}\left(\zeta_{\varepsilon_{n}}\right) \geq$ $\left\langle A_{\varepsilon_{n}}, h\right\rangle \mathrm{e}^{-\lambda\left(\widetilde{X}\left(\zeta_{\varepsilon_{n}}\right)+c_{\lambda} \zeta_{\varepsilon_{n}}\right)}$; thus, by Lemma 8 , it suffices to show

$$
\widetilde{Q}_{0 y}^{\lambda}\left(\limsup _{n \rightarrow+\infty}\left\langle A_{\varepsilon_{n}}, h\right\rangle \mathrm{e}^{-\lambda\left(\widetilde{X}\left(\zeta_{\varepsilon_{n}}\right)+c_{\lambda} \zeta_{\varepsilon_{n}}\right)}=+\infty\right)=1 .
$$

Obviously we have

$$
\left\langle A_{\varepsilon_{n}}, h\right\rangle \mathrm{e}^{-\lambda\left(\widetilde{X}\left(\zeta_{\varepsilon_{n}}\right)+c_{\lambda} \zeta_{\varepsilon_{n}}\right)}=\exp \left\{n\left[\frac{\log \left\langle A_{\varepsilon_{n}}, h\right\rangle}{n}-\lambda \frac{\zeta_{\varepsilon_{n}}}{n}\left(\frac{\widetilde{X}\left(\zeta_{\varepsilon_{n}}\right)}{\zeta_{\varepsilon_{n}}}+c_{\lambda}\right)\right]\right\} .
$$

Note that $\widetilde{Q}_{0 y}^{\lambda}\left(\lim _{t \rightarrow+\infty} \widetilde{X}(t) / t+c_{\lambda}=c_{\lambda}-\lambda>0\right)=1$, since $\tilde{X}$ moves as a Brownian motion with drift $-\lambda$ under $\widetilde{Q}_{0 y}^{\lambda}$. In addition, by the strong law of large numbers we have

$$
\widetilde{Q}_{0 y}^{\lambda}\left(\limsup _{n \rightarrow+\infty} \frac{\zeta_{\varepsilon_{n}}}{n} \leq \sum_{k \in S}\left(a_{k}+\lambda^{*}\right)<+\infty\right)=1 .
$$


Therefore, to prove (14), we only need to prove

$$
\widetilde{Q}_{0 y}^{\lambda}\left(\limsup _{n \rightarrow+\infty} \frac{\log \left\langle A_{\varepsilon_{n}}, h\right\rangle}{n}=+\infty\right)=1 .
$$

Let $N_{i}(n)$ denote the total number of jumps of $\tilde{Y}$ before it hits state $i$ for the $n$th time. Since $\widetilde{Y}$ moves as an irreducible Markov chain under $\widetilde{Q}_{0 y}^{\lambda}, n / N_{i}(n)$ converges to a positive constant with probability one. Note that $\left\{A_{\varepsilon_{N_{i}(n)}}: n \geq 0\right\}$ is a sequence of independent random vectors with the same distribution law $\left\{\hat{p}_{k}(i): k \in \mathbb{Z}_{+}^{d}\right\}$. The moment condition on $\xi_{i j}$ implies that $\widetilde{Q}_{0 y}^{\lambda} \log \left\langle A_{\varepsilon_{N_{i}(n)}}, h\right\rangle=+\infty$. It follows from the Borel-Cantelli lemma that $\widetilde{Q}_{0 y}^{\lambda}\left(\lim \sup _{n \rightarrow+\infty} \log \left\langle A_{\varepsilon_{N_{i}(n)}}, h\right\rangle / n=+\infty\right)=1$ and, consequently,

$$
\widetilde{Q}_{0 y}^{\lambda}\left(\limsup _{n \rightarrow+\infty} \frac{\log \left\langle A_{\varepsilon_{N_{i}(n)}}, h\right\rangle}{N_{i}(n)}=+\infty\right)=1,
$$

which implies (15).

Now we suppose that $E\left(\xi_{i j} \log ^{+} \xi_{i j}\right)<+\infty$ for all $i, j \in S$. Then, for every $i \in S$ we have $\widetilde{Q}_{0 y}^{\lambda}\left(\lim \sup _{n \rightarrow+\infty} \log \left\langle A_{\varepsilon_{N_{i}}(n)}, h\right\rangle / N_{i}(n)=0\right)=1$ and, consequently,

$$
\begin{aligned}
& \widetilde{Q}_{0 y}^{\lambda}\left(\sum_{n=1}^{+\infty}\left\langle A_{\varepsilon_{n}}, h\right\rangle \mathrm{e}^{-\lambda\left(\widetilde{X}\left(\zeta_{\varepsilon_{n}}\right)+c_{\lambda} \zeta_{\varepsilon_{n}}\right)}<+\infty\right) \\
& \quad=\widetilde{Q}_{0 y}^{\lambda}\left(\sum_{i \in S} \sum_{n=1}^{+\infty}\left\langle A_{\varepsilon_{N_{i}(n)}}, h\right\rangle \mathrm{e}^{-\lambda\left(\widetilde{X}\left(\zeta_{\varepsilon_{N_{i}}(n)}\right)+c_{\lambda} \zeta_{\varepsilon_{N_{i}(n)}}\right)}<+\infty\right) \\
& \quad=\widetilde{Q}_{0 y}^{\lambda}\left(\sum_{i \in S} \sum_{n=1}^{+\infty} \exp \left\{N_{i}(n)\left(\frac{\log \left\langle A_{\varepsilon_{N_{i}}(n)}, h\right\rangle}{N_{i}(n)}-\lambda \frac{\zeta_{\varepsilon_{N_{i}(n)}}}{N_{i}(n)}\left(\frac{\tilde{X}\left(\zeta_{\varepsilon_{N_{i}(n)}}\right)}{\zeta_{\varepsilon_{N_{i}(n)}}}+c_{\lambda}\right)\right)\right\}<+\infty\right) \\
& \quad=1,
\end{aligned}
$$

where in the last equality we used the fact that $\tilde{X}\left(\zeta_{\varepsilon_{n}}\right) / \zeta_{\varepsilon_{n}} \rightarrow-\lambda>-c_{\lambda}$ as $n \rightarrow+\infty$. Therefore, the second term in (13) is bounded from above for all $t>0$. In addition, under $\widetilde{Q}_{0 y}^{\lambda},-\lambda\left(\widetilde{X}(t)+c_{\lambda} t\right)=-\lambda t\left(\widetilde{X}(t) / t+c_{\lambda}\right) \rightarrow-\infty$ as $t \rightarrow+\infty$. Thus, the first term in (13) is also bounded from above. So we have $\widetilde{Q}_{0 y}^{\lambda}\left(\lim \sup _{t \rightarrow+\infty} \widetilde{Q}_{0 y}^{\lambda}\left(W_{\lambda}(t) \mid \widetilde{\mathscr{Q}}\right)<+\infty\right)=1$, and then $\widetilde{Q}_{0 y}^{\lambda}\left(\lim \sup _{t \rightarrow+\infty} W_{\lambda}(t)<+\infty\right)=1$ by Fatou's lemma. Therefore, by Lemma $8, W_{\lambda}(t)$ converges to $W(\lambda)$ in $L^{1}\left(P_{0 y}\right)$ which implies that $W(\lambda)$ is nondegenerate.

Let $q_{y}:=P_{0 y}(W(\lambda)=0)<1$. For any $t>s \geq 0$, we have

$$
W_{\lambda}(t)=\sum_{v \in Z(s)} \mathrm{e}^{-\lambda\left(X_{v}(s)+c_{\lambda} s\right)} W_{\lambda}(t-s, v),
$$

where $\left\{W_{\lambda}(t-s, v), v \in Z(s)\right\}$ are independent copies of $W_{\lambda}(t-s)$ initiated by $v \in Z(s)$. We use $\sharp A$ to denote the cardinal of a finite set $A$. It follows that

$$
q_{y}=E_{0 y}\left(\prod_{v \in Z(s)} q_{Y_{v}}\right) \leq E_{0 y}\left(\left(\max _{j \in S} q_{j}\right)^{\sharp Z(s)}\right) .
$$

The Kesten-Stigum theorem for multitype Markov branching processes (see, for example, [1]) confirms that the total population size $\sharp Z(s)$ goes to infinity almost surely on the nonextinction set; thus, we have $q_{y}=0$ by the dominated convergence theorem. Hence, we complete the proof. 
Define $L(t):=\inf \left\{X_{u}(t): u \in Z(t)\right\}$, i.e. $L(t)$ denotes the position of the leftmost particle at time $t$. Then we have the following result.

Theorem 4. For any $(x, y) \in \mathbb{R} \times S, P_{x y}\left(\lim _{t \rightarrow+\infty} L(t)+\underline{c} t=+\infty\right)=1$. Moreover, if $E\left(\xi_{i j} \log ^{+} \xi_{i j}\right)<+\infty$ for all $i, j \in S$, then $P_{x y}\left(\lim _{t \rightarrow+\infty} L(t) / t=-\underline{c}\right)=1$.

Proof. It is sufficient to prove the conclusion under measure $P_{0 y}$. Note that

$$
W_{\lambda}(t) \geq C_{1} \mathrm{e}^{-\lambda\left(L(t)+c_{\lambda} t\right)}=C_{1} \mathrm{e}^{-\lambda t\left(L(t) / t+c_{\lambda}\right)},
$$

for some constant $C_{1}>0$. Since $\lim _{t \rightarrow+\infty} W_{\underline{\lambda}}(t)=0$, it follows from (16) that

$$
P_{0 y}\left(\lim _{t \rightarrow+\infty} L(t)+\underline{c} t=+\infty\right)=1 \quad \text { and } \quad P_{0 y}\left(\liminf _{t \rightarrow+\infty} \frac{L(t)}{t} \geq-\underline{c}\right)=1 .
$$

Recall that the spine moves as a Brownian motion with drift $-\lambda$ under the measure $\widetilde{Q}_{0 y}^{\lambda}$, so we have $\widetilde{Q}_{0 y}^{\lambda}\left(\lim _{t \rightarrow+\infty} \tilde{X}(t) / t=-\lambda\right)=1$. The proof of Theorem 3 shows that if $E\left(\xi_{i j} \log ^{+} \xi_{i j}\right)<$ $+\infty$ for all $i, j \in S$, then $\mathrm{d} Q_{0 y}^{\lambda}=W(\lambda) / h_{y} \mathrm{~d} P_{0 y}$ and $P_{0 y}(W(\lambda)>0)=1$ for any $\lambda \in(0, \lambda)$. This implies that $Q_{0 y}^{\lambda}(W(\lambda)>0)=1$ and $P_{0 y}$ is absolutely continuous with respect to $Q_{0 y}^{\lambda}$. Hence, for any $0<\lambda<\underline{\lambda}$,

$$
P_{0 y}\left(\limsup _{t \rightarrow+\infty} \frac{L(t)}{t} \leq-\lambda\right) \geq P_{0 y}\left(\lim _{t \rightarrow+\infty} \frac{\tilde{X}(t)}{t}=-\lambda\right)=1 .
$$

Thus, $P_{0 y}\left(\lim \sup _{t \rightarrow+\infty} L(t) / t \leq-\underline{\lambda}=-\underline{c}\right)=1$. We complete the proof.

Proof of Theorem 1(a). It follows from Theorem 3 that $w(x, y)$ is nontrivial and $\lim _{x \rightarrow-\infty}$ $w(x, y)=0$ since $P_{0 y}(W(\lambda)=0)=0$. By definition, it is clear that $\lim _{x \rightarrow+\infty} w(x, y)=1$, and that $x \mapsto w(x, y)$ is monotone for every $y \in S$. In addition,

$$
\begin{aligned}
w(x, y) & =E_{x y}\left[\exp \left\{-\sum_{v \in Z(s)} \lim _{t \rightarrow+\infty} \sum_{\substack{u \in Z(t) \\
v \prec u}} h_{Y_{u}} \mathrm{e}^{-\lambda\left(X_{u}(t)+c t\right)}\right\}\right] \\
& =E_{x y}\left[\prod_{v \in Z(s)} E_{X_{v}(s) Y_{v}}\left(\exp \left\{-\mathrm{e}^{-\lambda c s} \lim _{t \rightarrow+\infty} \sum_{u \in Z(t-s)} h_{Y_{u}} \mathrm{e}^{-\lambda\left(X_{u}(t-s)+c(t-s)\right)}\right\}\right)\right] \\
& =E_{x y}\left[\prod_{v \in Z(s)} w\left(X_{v}(s)+c s, Y_{v}\right)\right]
\end{aligned}
$$

Thus, it follows from Lemma 6 that $u(t, x, y):=w(x-c t, y)$ is a traveling wave solution to (1) with wave speed $c$. Since $\lim _{x \rightarrow+\infty} w(x, y)=1$ and $E_{0 y} W(\lambda)=E_{0 y} W_{\lambda}(0)=h_{y}$,

$$
\frac{1-w(x, y)}{h_{y} \mathrm{e}^{-\lambda x}}=\frac{1-E_{0 y}\left[\exp \left\{-\mathrm{e}^{-\lambda x} W(\lambda)\right\}\right]}{E_{0 y}\left[\mathrm{e}^{-\lambda x} W(\lambda)\right]} \rightarrow 1 \quad \text { as } x \rightarrow+\infty .
$$

The rest of this proof is dedicated to the uniqueness. We consider the space-time barrier $\Gamma^{\left(x, c_{\lambda}\right)}:=\left\{(y, t) \in \mathbb{R} \times \mathbb{R}^{+}: y+c_{\lambda} t=x\right\}$ for $x \geq 0$. By arresting lines of descendants the first time they hit this barrier, we produce a random collection of particles $C\left(x, c_{\lambda}\right)=$ $\bigcup_{i \in S} C_{i}\left(x, c_{\lambda}\right)$, where $C_{i}\left(x, c_{\lambda}\right)$ denotes the subset of type $i$ particles. Here, $\left\{C\left(x, c_{\lambda}\right): x \geq 0\right\}$ is a family of stopping lines. We say that $\left\{C\left(x, c_{\lambda}\right): x \geq 0\right\}$ is dissecting in the sense that all lines 
of descendants will hit $\Gamma^{\left(x, c_{\lambda}\right)}$ with probability one for all $x>0$ because $\lim _{t \rightarrow+\infty} L(t)+c t=$ $+\infty$ for $c \geq \underline{c}$. We also observe that $\left\{C\left(x, c_{\lambda}\right): x \geq 0\right\}$ is tending to infinity in the sense that, for each $n \in \mathbb{N}$, we can choose $x$ sufficiently large such that particles in $C\left(x, c_{\lambda}\right)$ are descendants of the $n$th generation. (For more information on general stopping lines and properties of them, we refer to [4] and [9].) Let $\mathcal{F}_{\Gamma^{\left(x, c_{\lambda}\right)}}$ be the natural filtration generated by ancestral type and spatial paths receding from particles at the moment they hit $\Gamma^{\left(x, c_{\lambda}\right)}$. Let $\Phi_{c_{\lambda}}$ be an arbitrary traveling wave at speed $c_{\lambda}$. Then

$$
\begin{aligned}
M_{x}\left(z, c_{\lambda}\right) & :=\prod_{u \in C\left(x, c_{\lambda}\right)} \Phi_{c_{\lambda}}\left(z+X_{u}(t)+c_{\lambda} t, Y_{u}\right) \\
& =\exp \left\{\sum_{i \in S} \sharp C_{i}\left(x, c_{\lambda}\right) \log \Phi_{c_{\lambda}}(z+x, i)\right\}
\end{aligned}
$$

is a $P_{0 y}$-martingale with respect to $\left\{\mathcal{F}_{\Gamma^{\left(x, c_{\lambda}\right)}}: x \geq 0\right\}$. It converges to $\Phi_{c_{\lambda}}(z, \lambda)$ almost surely and in $L^{1}\left(P_{0 y}\right)$ (by boundedness), and then it follows that

$$
\lim _{x \rightarrow+\infty}-\sum_{i \in S} \sharp C_{i}\left(x, c_{\lambda}\right) \log \Phi_{c_{\lambda}}(z+x, i)
$$

exists and is positive with positive probability.

Obviously, for any $x_{2}>x_{1} \geq 0$ and any $v \in C\left(x_{2}, c_{\lambda}\right)$, there exists a unique $u \in C\left(x_{1}, c_{\lambda}\right)$ such that $u \prec v$. In fact, $\left\{\left(\sharp C_{1}\left(x, c_{\lambda}\right), \ldots, \sharp C_{d}\left(x, c_{\lambda}\right)\right)^{\top}: x \geq 0\right\}$ forms a continuous-time multitype Markov branching process ( $x$ plays the role of time). This follows from the strong Markov branching property (see, for example, [9]). Moreover, it follows from the fact $P_{0 y}\left(\lim _{t \rightarrow+\infty} \widetilde{X}(t)+c_{\lambda} t=+\infty\right)$ and the irreducibility of $\widetilde{Y}$ that $\left\{\left(\sharp C_{1}\left(x, c_{\lambda}\right), \ldots, \sharp C_{d}\left(x, c_{\lambda}\right)\right)^{\top}: x \geq 0\right\}$ is nonextinct and positive regular. Let $M_{c_{\lambda}}(x)=$ $\left(m_{i j}^{c_{\lambda}}(x)\right)_{i, j \in S}$ where $m_{i j}^{c}(x)=E_{0 i} \sharp C_{j}\left(x, c_{\lambda}\right)$, and let $A_{c_{\lambda}}$ be the matrix such that $M_{c_{\lambda}}(x)=$ $e^{A_{c_{\lambda} x}}$. By the Perron-Frobenius theorem, we can find a simple positive eigenvalue $\lambda_{c_{\lambda}}^{*}$ of $A_{c_{\lambda}}$, and corresponding positive left and right eigenvectors $\pi_{c_{\lambda}}=\left(\pi_{c_{\lambda}}^{1}, \ldots, \pi_{c_{\lambda}}^{d}\right)^{\top}$ and $h_{c_{\lambda}}=$ $\left(h_{c_{\lambda}}^{1}, \ldots, h_{c_{\lambda}}^{d}\right)^{\top}$ such that $\left\langle\pi_{c_{\lambda}}, h_{c_{\lambda}}\right\rangle=\left\langle\pi_{c_{\lambda}}, 1\right\rangle=1$. Immediately,

$$
\sum_{j \in S} m_{i j}^{c_{\lambda}}(x) h_{c_{\lambda}}^{j} \mathrm{e}^{-\lambda_{c_{\lambda}}^{*} x}=h_{c_{\lambda}}^{i}, \quad \text { for all } i \in S
$$

For $x \geq 0$, define

$$
W_{\Gamma^{\left(x, c_{\lambda}\right)}}(\lambda):=\sum_{u \in C\left(x, c_{\lambda}\right)} h_{Y_{u}} \mathrm{e}^{-\lambda\left(X_{u}(t)+c_{\lambda} t\right)}=\sum_{i \in S} \sharp C_{i}\left(x, c_{\lambda}\right) h_{i} \mathrm{e}^{-\lambda x} .
$$

Then $\left\{W_{\Gamma\left(x, c_{\lambda}\right)}(\lambda): x \geq 0\right\}$ is a $P_{0 y}$-martingale with respect to $\left\{\mathcal{F}_{\Gamma^{\left(x, c_{\lambda}\right)}}: x \geq 0\right\}$ and, consequently,

$$
\sum_{j \in S} m_{i j}^{c \lambda}(x) h_{j} \mathrm{e}^{-\lambda x}=h_{i}, \quad \text { for all } i \in S
$$

in other words, $\mathrm{e}^{\lambda x}$ is an eigenvalue of $M_{c_{\lambda}}(x)$ with corresponding right eigenvector $h$. Using similar arguments as in [11, Theorem 8], we can show that

$$
\lim _{x \rightarrow+\infty} \sum_{i \in S} \sharp C_{i}\left(x, c_{\lambda}\right) h_{i} \mathrm{e}^{-\lambda x}=W(\lambda), \quad P_{0 y} \text {-almost surely and in } L^{1}\left(P_{0 y}\right) .
$$


On the other hand, by the Kensten-Stigum theorem (see, for example, [6, Theorem 2.1]) we have, for any $i \in S$,

$$
\lim _{x \rightarrow+\infty} \sharp C_{i}\left(x, c_{\lambda}\right) \mathrm{e}^{-\lambda_{c_{\lambda}}^{*} x}=\pi_{c_{\lambda}}^{i} W_{c_{\lambda}}, \quad P_{0 y} \text {-almost surely, }
$$

where $W_{c_{\lambda}}=\lim _{x \rightarrow+\infty} \sum_{i \in S} \sharp C_{i}\left(x, c_{\lambda}\right) \pi_{c_{\lambda}}^{i} \mathrm{e}^{-\lambda_{c_{\lambda}}^{*} x}<+\infty$. Combining (20) and (21), we conclude that $\lambda_{c_{\lambda}}^{*}=\lambda$ and $P_{0 y}\left(W_{c_{\lambda}}=\alpha W(\lambda)\right)=1$ for some constant $\alpha>0$. Using (18) and (19), we get $h_{c_{\lambda}}=\alpha h$. Thus, by (21) we have, for any $i \in S$,

$$
\lim _{x \rightarrow+\infty} \sharp C_{i}\left(x, c_{\lambda}\right) \mathrm{e}^{-\lambda x}=\alpha \pi_{c_{\lambda}}^{i} W(\lambda), \quad P_{0 y} \text {-almost surely. }
$$

It follows from (17) and (22) that $\beta:=\lim _{x \rightarrow+\infty}-\alpha \sum_{i \in S} \pi_{c_{\lambda}}^{i} \mathrm{e}^{\lambda x} \log \Phi_{c_{\lambda}}(x, i)$ exists and is positive. Uniqueness (up to a multiplicative constant) is now immediate since

$$
\begin{aligned}
\Phi_{c_{\lambda}}(z, y) & =E_{0 y}\left(\lim _{x \rightarrow+\infty} M_{x}\left(z, c_{\lambda}\right)\right) \\
& =E_{0 y}\left(\exp \left\{\lim _{x \rightarrow+\infty} \sum_{i \in S} \sharp C_{i}\left(x, c_{\lambda}\right) \log \Phi_{c_{\lambda}}(z+x, i)\right\}\right) \\
& =E_{0 y}\left(\exp \left\{\lim _{x \rightarrow+\infty} \alpha \sum_{i \in S} \pi_{c_{\lambda}}^{i} \mathrm{e}^{\lambda x} W(\lambda) \log \Phi_{c_{\lambda}}(z+x, i)\right\}\right) \\
& =E_{0 y}\left(\exp \left\{-\beta W(\lambda) \mathrm{e}^{-\lambda z}\right\}\right) .
\end{aligned}
$$

Hence, we complete the proof.

Proof of Theorem $1(b)$. We assume that $w(x, y)$ provides a monotone traveling wave solution to (1) with speed $c<\underline{c}$. Then, by Lemma $6, \prod_{u \in Z(t)} w\left(X_{u}(t)+x+c t, Y_{u}\right)$ is a bounded martingale under $P_{0 y}$. It converges almost surely and in $L^{1}\left(P_{0 y}\right)$ to some random variable. On the other hand, since $0 \leq w(x, y) \leq 1$ and $\lim _{t \rightarrow \infty} L(t)+c t=-\infty$, we have

$$
\prod_{u \in Z(t)} w\left(X_{u}(t)+x+c t, Y_{u}\right) \leq w\left(L(t)+c t, Y_{L}(t)\right) \rightarrow 0
$$

where $Y_{L}(t)$ denotes the type of leftmost particle at time $t$. Thus, $w(x, y) \equiv 0$ which contradicts the assumption.

\section{Proof of Theorem 2}

Note that $M_{t}(\lambda)$ defined in (3) is a signed martingale and therefore it does not necessarily converge almost surely. A technique used by Kyprianou [11] to get round this problem in the case of a single-type branching Brownian motion is to consider a truncated form of the derivative martingale which turns out to be a positive martingale. In order to describe the aforementioned martingale for MBBM we need more notation and lemmas.

Lemma 9. (Kyprianou [11, Section 5].) Suppose that $B=\left\{B_{t}: t \geq 0\right\}$ is a standard Brownian motion on $\mathbb{R}$ with law $\mathbb{B}$ and natural filtration $\left\{\mathcal{L}_{t}, t \geq 0\right\}$. For any $z>0$, define $\tau_{\lambda}:=\inf \{t>$ $\left.0: z+B_{t}+\lambda t \leq 0\right\}$, then

$$
m_{\lambda}(t):=\frac{z+B_{t}+\lambda t}{z} \mathrm{e}^{-\lambda\left(B_{t}+\lambda t / 2\right)} \mathbf{1}_{\left\{t<\tau_{\lambda}\right\}}
$$


is a positive mean-one martingale. Define another probability measure $\hat{\mathbb{B}}_{z}^{\lambda}$ by $\left.\mathrm{d} \hat{\mathbb{B}}_{z}^{\lambda}\right|_{\mathscr{L}_{t}}=$ $\left.m_{\lambda}(t) \mathrm{d} \mathbb{B}\right|_{\mathscr{L}_{t}}$. Then, under measure $\hat{\mathbb{B}}_{z}^{\lambda},\left\{z+B_{t}+\lambda t: t \geq 0\right\}$ is a standard Bessel-3 process starting from $z$.

Define the space-time barrier $\Gamma^{(-z, \lambda)}:=\left\{(y, t) \in \mathbb{R} \times \mathbb{R}^{+}: y+\lambda t=-z\right\}$ for $z \geq 0$. Here, $\widetilde{Z}(t)$ denotes the subset of $Z(t)$ consisting of all particles which are alive at $t$ and have ancestry (including themselves) whose spatial paths have not met $\Gamma^{(-z, \lambda)}$ by time $t$. From the many-to-one formula, we see that

$$
V_{\lambda}(t):=\sum_{u \in \widetilde{Z}(t)} h_{Y_{u}}\left(z+X_{u}(t)+\lambda t\right) \mathrm{e}^{-\lambda\left(X_{u}(t)+c_{\lambda} t\right)}
$$

is a nonnegative martingale. We want to define a new probability measure $\widetilde{R}_{0 y}^{\lambda}$ such that, if $R_{0 y}^{\lambda}:=\widetilde{R}_{0 y}^{\lambda} \mid \mathcal{F}$, then

$$
\left.\mathrm{d} R_{0 y}^{\lambda}\right|_{\mathscr{F}_{t}}=\left.\frac{V_{\lambda}(t)}{V_{\lambda}(0)} \mathrm{d} P_{0 y}\right|_{\mathscr{F}_{t}}, \quad \text { for all } t>0
$$

To this end, $\tilde{R}_{0 y}^{\lambda}$ should have the following decomposition:

$$
\begin{aligned}
\left.\mathrm{d} \widetilde{R}_{0 y}^{\lambda}(\tau, M, \varepsilon)\right|_{\widetilde{F}_{t}}= & \mathrm{d} \hat{\mathbb{P}}_{y}(\tilde{Y}) \mathrm{d} \hat{\mathbb{B}}_{z}^{\lambda}(\tilde{X}) \prod_{v \prec \varepsilon_{n_{t}}} \frac{\hat{p}_{A_{v}}\left(Y_{v}\right)}{\widehat{P}\left(Y_{v}, Y_{v+1}\right)} \frac{A_{v}\left(Y_{v+1}\right) h_{Y_{v+1}}}{\left\langle A_{v}, h\right\rangle} \\
& \times \prod_{v<\varepsilon_{n_{t}}}\left[\frac{1}{A_{v}\left(Y_{v+1}\right)} \prod_{j: v j \in O_{v}} \mathrm{~d} P_{\widetilde{X}_{\zeta v}}^{t-\zeta_{v j}}\left((\tau, M)_{j}^{v}\right)\right] .
\end{aligned}
$$

Remark 2. Under $\widetilde{R}_{0 y}^{\lambda}$, the spine's spatial process $\widetilde{X}$ satisfies that $\{z+\widetilde{X}(t)+\lambda t: t \geq 0\}$ is a Bessel-3 process starting from $z$. Therefore, it never meets the barrier $\Gamma^{(-z, \lambda)}$.

Put

$$
M_{\lambda}(t):=\sum_{u \in Z(t)} h_{Y_{u}}\left(z+X_{u}(t)+\lambda t\right) \mathrm{e}^{-\lambda\left(X_{u}(t)+c_{\lambda} t\right)} .
$$

If we can prove that $M_{\lambda}(t)$ converges to a nondegenerate limit, similar analysis as in the supercritical case can be carried out to obtain traveling wave solutions of (1). For this purpose, we need the following lemma.

Lemma 10. Let $V(\lambda)=\lim _{t \rightarrow+\infty} V_{\lambda}(t)$. For any $\lambda \geq \underline{\lambda}, \lim _{t \rightarrow+\infty} M_{\lambda}(t)$ exists and is equivalent to $V(\lambda)$ almost surely under $P_{0 y}$. In addition, $M(\lambda):=\lim _{t \rightarrow+\infty} M_{\lambda}(t)$ does not depend on $z$.

Proof. Recall that $V_{\lambda}(t)$ is a nonnegative martingale, its limit $V(\lambda)$ exists almost surely. Let $\gamma^{(-z, \lambda)}$ denote the event that the MBBM remains entirely to the right of $\Gamma^{(-z, \lambda)}$, then

$$
\lim _{t \rightarrow+\infty} M_{\lambda}(t)=\lim _{t \rightarrow+\infty} V_{\lambda}(t), \quad \text { on } \gamma^{(-z, \lambda)}, \quad P_{0 y} \text {-almost surely. }
$$

Since $P_{0 y}\left(\lim _{t \rightarrow+\infty} L(t)+\underline{c} t=+\infty\right)=1$, we have $P_{0 y}\left(\inf _{t \geq 0}\{L(t)+\lambda t\}>-\infty\right)=1$ for all $\lambda \geq \underline{\lambda}$. Thus, $P_{0 y}\left(\gamma^{(-z, \lambda)}\right)=P_{0 y}\left(\inf _{t \geq 0}\{L(t)+\lambda t\}>-z\right) \uparrow 1$ as $z \uparrow+\infty$. Therefore, we have $P_{0 y}\left(\lim _{t \rightarrow+\infty} M_{\lambda}(t)=\lim _{t \rightarrow+\infty} V_{\lambda}(t)\right)=1$, which implies that, for every $\lambda \geq \underline{\lambda}$, $\lim _{t \rightarrow+\infty} M_{\lambda}(t)$ exists and is equal to $V(\lambda) P_{0 y}$-almost surely. Note that

$$
M_{\lambda}(t)=\sum_{u \in Z(t)} h_{Y_{u}}\left(X_{u}(t)+\lambda t\right) \mathrm{e}^{-\lambda\left(X_{u}(t)+c_{\lambda} t\right)}+z W_{\lambda}(t) .
$$


By Theorem 3, the second term of the right-hand side converges to 0 for $\lambda \geq \underline{\lambda}$; hence, the limit $M(\lambda)$ does not depend on $z$. Hence, we complete the proof.

Next,we focus on the limit theorem for the martingale $V_{\underline{\lambda}}(t)$. Hereafter, we simply write $\widetilde{R}_{0 y}^{\lambda}$ as $\widetilde{R}_{0 y}$.

Theorem 5. Suppose that $\lambda=\underline{\lambda}$.

1. If $E \xi_{i j}\left(\log ^{+} \xi_{i j}\right)^{2}=+\infty$ for some $i, j \in S$, then $V(\underline{\lambda})=0 P_{x y}$-almost surely.

2. If $E \xi_{i j}\left(\log ^{+} \xi_{i j}\right)^{2}<+\infty$ for all $i, j \in S$, then $V_{\underline{\lambda}}(t)$ converges to $V(\underline{\lambda})$ in $L^{1}\left(P_{x y}\right)$ and $P_{x y}(V(\underline{\lambda})=0)=0$.

To prove Theorem 5, we first prove some lemmas.

Lemma 11. We have the following spine decomposition for $V_{\underline{\lambda}}(t)$ :

$$
\begin{aligned}
\widetilde{R}_{0 y}\left(V_{\underline{\lambda}}(t) \mid \tilde{\mathcal{g}}\right)= & h_{\widetilde{Y}_{t}}(z+\tilde{X}(t)+\underline{\lambda}) \mathrm{e}^{-\underline{\lambda}(\tilde{X}(t)+\underline{c} t)} \\
& +\sum_{j \in S} \sum_{v \prec \varepsilon_{n_{t}}}\left(A_{v}(j)-\delta_{Y_{v+1} j}\right) h_{j}\left(z+\tilde{X}\left(\zeta_{v}\right)+\underline{\lambda} t\right) \mathrm{e}^{-\underline{\lambda}\left(\widetilde{X}\left(\zeta_{v}\right)+\underline{c} \zeta_{v}\right)}
\end{aligned}
$$

Lemma 12. (a) If $E \xi_{i j}\left(\log ^{+} \xi_{i j}\right)^{2}=+\infty$ for some $i, j \in S$, then

$$
\limsup _{n \rightarrow+\infty}\left\langle A_{\varepsilon_{n}}, h\right\rangle\left(z+\tilde{X}\left(\zeta_{\varepsilon_{n}}\right)+\underline{\lambda} \zeta_{\varepsilon_{n}}\right) \mathrm{e}^{-\underline{\lambda}\left(\tilde{X}\left(\zeta_{\varepsilon_{n}}\right)+\underline{c} \zeta_{\varepsilon_{n}}\right)}=+\infty, \quad \widetilde{R}_{0 y} \text {-almost surely. }
$$

(b) If $E \xi_{i j}\left(\log ^{+} \xi_{i j}\right)^{2}<+\infty$ for all $i, j \in S$, then

$$
\sum_{n=0}^{+\infty}\left\langle A_{\varepsilon_{n}}, h\right\rangle\left(z+\tilde{X}\left(\zeta_{\varepsilon_{n}}\right)+\underline{\lambda} \zeta_{\varepsilon_{n}}\right) \mathrm{e}^{-\underline{\lambda}\left(\tilde{X}\left(\zeta_{\varepsilon_{n}}\right)+\underline{c} \zeta_{\varepsilon_{n}}\right)}<+\infty, \quad \widetilde{R}_{0 y} \text {-almost surely }
$$

Proof. (a) We want to show that, for any $M \in(0,+\infty)$,

$$
\sum_{n=0}^{+\infty} \mathbf{1}_{\left\{\left\langle A_{\varepsilon_{n}}, h\right\rangle\left(z+\widetilde{X}\left(\zeta_{\varepsilon_{n}}\right)+\underline{\lambda} \zeta_{\varepsilon_{n}}\right) \mathrm{e}^{\left.-\underline{\lambda}\left(\tilde{X}\left(\zeta \varepsilon_{n}\right)+\underline{c} \xi_{\varepsilon_{n}}\right) \geq M\right\}}\right.}=+\infty, \quad \widetilde{R}_{0 y} \text {-almost surely. }
$$

For any set $B \in \mathscr{B}[0, \infty) \times \mathscr{B}\left(\mathbb{Z}_{+}^{d}\right)$, define $\phi(B)=\#\left\{n \geq 0:\left(\zeta_{\varepsilon_{n}}, A_{\varepsilon_{n}}\right) \in B\right\}$. Then, conditioned on $g_{\widetilde{Y}}^{\widetilde{Y}}, \phi$ is a Poisson random measure on $[0, \infty) \times \overline{\mathbb{Z}}_{+}^{d}$ with intensity $\left(a_{\widetilde{Y}_{t}}+\lambda^{*}\right) \mathrm{d} t$ $\times \sum_{k \in \mathbb{Z}_{+}^{d}} \hat{p}_{k}\left(\widetilde{Y}_{t}\right) \delta_{k}(\mathrm{~d} y)$ (here, $\delta$ denotes the delta function). Thus, for any $T \in(0, \infty)$, given $g^{\widetilde{Y}}, \#\left\{n \geq 0: \zeta_{\varepsilon_{n}} \leq T,\left\langle A_{\varepsilon_{n}}, h\right\rangle\left(z+\widetilde{X}\left(\zeta_{\varepsilon_{n}}\right)+\underline{\lambda} \zeta_{\varepsilon_{n}}\right) \mathrm{e}^{-\underline{\lambda}\left(\widetilde{X}\left(\zeta_{\varepsilon_{n}}\right)+\underline{c} \zeta_{\varepsilon_{n}}\right)} \geq M\right\}$ is a Poisson random variable with parameter

$$
\int_{0}^{T}\left(a_{\widetilde{Y}_{t}}+\lambda^{*}\right) \sum_{k \in \mathbb{Z}_{+}^{d}} \hat{p}_{k}\left(\widetilde{Y}_{t}\right) \mathbf{1}_{\left\{\langle k, h\rangle(z+\widetilde{X}(t)+\underline{\lambda} t) \mathrm{e}^{-\underline{\lambda}(\tilde{X}(t)+\underline{c} t) \geq M\}}\right.} \mathrm{d} t .
$$


Hence, to prove (23), we only need to show that

$$
\int_{0}^{+\infty}\left(a_{\widetilde{Y}_{t}}+\lambda^{*}\right) \sum_{k \in \mathbb{Z}_{+}^{d}} \hat{p}_{k}\left(\tilde{Y}_{t}\right) \mathbf{1}_{\left\{\langle k, h\rangle(z+\widetilde{X}(t)+\underline{\lambda} t) \mathrm{e}^{-\underline{\lambda}(\tilde{X}(t)+\underline{c} t) \geq M\}}\right.} \mathrm{d} t=+\infty, \quad \widetilde{R}_{0 y} \text {-almost surely. }
$$

Since $\min \left\{a_{l}: l \in S\right\}>0$, it is sufficient to prove that

$$
\widetilde{R}_{0 y}\left(\int_{0}^{+\infty} \sum_{k \in \mathbb{Z}_{+}^{d}} \hat{p}_{k}\left(\widetilde{Y}_{t}\right) \mathbf{1}_{\left\{\langle k, h\rangle(z+\tilde{X}(t)+\underline{\lambda} t) \mathrm{e}^{-\underline{\lambda}(\tilde{X}(t)+\underline{c} t) \geq M\}}\right.} \mathrm{d} t<+\infty\right)=0 .
$$

For any constant $c \in(0,+\infty)$, put

$$
E_{c}:=\left\{\int_{0}^{+\infty} \sum_{k \in \mathbb{Z}_{+}^{d}} \hat{p}_{k}\left(\tilde{Y}_{t}\right) \mathbf{1}_{\left\{\langle k, h\rangle(z+\widetilde{X}(t)+\underline{\lambda} t) \mathrm{e}^{-\underline{\lambda}(\tilde{X}(t)+\underline{c} t) \geq M\}}\right.} \mathrm{d} t<c\right\} .
$$

It suffices to show that $\widetilde{R}_{0 y}\left(E_{c}\right)=0$. In fact, we have

$$
\begin{aligned}
& c \geq \widetilde{R}_{0 y}\left(\mathbf{1}_{E_{c}} \int_{0}^{+\infty} \sum_{k \in \mathbb{Z}_{+}^{d}} \hat{p}_{k}\left(\widetilde{Y}_{t}\right) \mathbf{1}_{\left\{\langle k, h\rangle(z+\widetilde{X}(t)+\underline{\lambda} t) \mathrm{e}^{-\underline{\lambda}(\tilde{X}(t)+\underline{c} t) \geq M\}}\right.} \mathrm{d} t\right) \\
& =\int_{0}^{+\infty} \sum_{l \in S} \hat{\mathbb{P}}_{y}\left(\tilde{Y}_{t}=l\right) \sum_{k \in \mathbb{Z}_{+}^{d}} \hat{p}_{k}(l) \widetilde{R}_{0 y}\left(\mathbf{1}_{E_{c}} \mathbf{1}_{\left\{\operatorname{Bes}(t) \mathrm{e}^{-\lambda} \underline{\operatorname{Bes}(t)} \geq M\langle k, h\rangle^{-1} \mathrm{e}^{-\lambda} \underline{\lambda}\right\}}\right) \mathrm{d} t \\
& \geq \int_{0}^{+\infty} \hat{\mathbb{P}}_{y}\left(\tilde{Y}_{t}=i\right) \sum_{k \in \mathbb{Z}_{+}^{d}} \hat{p}_{k}(i) \widetilde{R}_{0 y}\left(\mathbf{1}_{E_{c}} \mathbf{1}_{\left\{\operatorname{Bes}(t) \mathrm{e}^{-\underline{\lambda} \operatorname{Bes}(t)} \geq M\langle k, h\rangle^{-1} \mathrm{e}^{-\underline{\lambda} z}\right\}}\right) \mathrm{d} t,
\end{aligned}
$$

where $\operatorname{Bes}(t):=z+\widetilde{X}(t)+\underline{\lambda} t$. It is known that, under $\hat{\mathbb{P}}_{y}, \widetilde{Y}$ moves as a Q-process with the invariant distribution $\tilde{\pi}_{l}=h_{l} \bar{\pi}_{l}$ for every $l \in S$. Consequently, there exists some $T>0$ such that, for any $t \geq T, \hat{\mathbb{P}}_{y}\left(\widetilde{Y}_{t}=i\right) \geq \frac{1}{2} \tilde{\pi}_{i}>0$. We continue the above domination:

$$
\begin{aligned}
c & \geq \frac{1}{2} \tilde{\pi}_{i} \sum_{k \in \mathbb{Z}_{+}^{d}} \hat{p}_{k}(i) \int_{T}^{+\infty} \widetilde{R}_{0 y}\left(\mathbf{1}_{E_{c}} \mathbf{1}_{\left\{\operatorname{Bes}(t) \mathrm{e}^{-\underline{\lambda} \operatorname{Bes}(t)} \geq M\langle k, h\rangle^{-1} \mathrm{e}^{-\underline{\lambda} z}\right\}}\right) \mathrm{d} t \\
& \geq \frac{1}{2} \tilde{\pi}_{i}\left(\sum_{k \in \mathbb{Z}_{+}^{d}} \hat{p}_{k}(i) \int_{0}^{+\infty} \widetilde{R}_{0 y}\left(\mathbf{1}_{E_{c}} \mathbf{1}_{\left\{\operatorname{Bes}(t) \mathrm{e}^{-\underline{\lambda} \operatorname{Bes}(t)} \geq M\langle k, h\rangle^{-1} \mathrm{e}^{-\underline{\lambda} z}\right\}}\right) \mathrm{d} t-T\right) .
\end{aligned}
$$

We consider a process $\left(\left(Q_{t}, W_{t}\right), \mathbb{P}\right)$ such that $\left\{Q_{t}, t \geq 0\right\}$ and $\left\{W_{t}, t \geq 0\right\}$ are independent, $\left(Q_{t}, \mathbb{P}\right)$ is identically distributed as $\left(\widetilde{Y}_{t}, \widetilde{R}_{0 y}\right)$, and $\left(W_{t}, \mathbb{P}\right)$ is a standard Brownian motion on $\mathbb{R}^{3}$ starting from 0 . Suppose that $\hat{z}$ is a point in $\mathbb{R}^{3}$ with norm $z$. It is known that $\left(\operatorname{Bes}(t), \widetilde{R}_{0 y}\right)$ is a Bessel-3 process starting from $z$, which is identically distributed as $\left(\left|W_{t}+\hat{z}\right|, \mathbb{P}\right)$, here $|\cdot|$ denotes the Euclidean norm. We still use $E_{c}$ to denote the counterpart set of $E_{c}$ with respect to $\left(\left(Q_{t}, W_{t}\right), \mathbb{P}\right)$. Immediately, we have

$$
\widetilde{R}_{0 y}\left(\mathbf{1}_{E_{c}} \mathbf{1}_{\left\{\operatorname{Bes}(t) \mathrm{e}^{-\underline{\lambda} \operatorname{Bes}(t)} \geq M\langle k, h\rangle^{-1} \mathrm{e}^{-\underline{\lambda} z}\right\}}\right)=\mathbb{P}\left(\mathbf{1}_{E_{c}} \mathbf{1}_{\left\{\left|W_{t}+\hat{z}\right| \mathrm{e}^{-\underline{\lambda}\left|W_{t}+\hat{z}\right|} \mid M\langle k, h\rangle^{-1} \mathrm{e}^{-\underline{\lambda} z}\right\}}\right)
$$


and $\widetilde{R}_{0 y}\left(E_{c}\right)=\mathbb{P}\left(E_{c}\right)$. We claim that there exists $K^{*}>0$ such that, when $|k| \geq K^{*}$,

$$
\left\{y \in \mathbb{R}^{3}: 1+z \leq|y| \leq \frac{\log ^{+}\langle k, h\rangle}{2 \underline{\lambda}}\right\} \subset\left\{y \in \mathbb{R}^{3}:|y+\hat{z}| \mathrm{e}^{-\underline{\lambda}|y+\hat{z}|} \geq M\langle k, h\rangle^{-1} \mathrm{e}^{-\underline{\lambda} z}\right\},
$$

which can be proved using basic analysis. Now we continue the estimation of (24) as follows:

$$
\begin{aligned}
& c \geq \frac{1}{2} \tilde{\pi}_{i}\left(\sum_{k \in \mathbb{Z}_{+}^{d}} \hat{p}_{k}(i) \int_{0}^{+\infty} \mathbb{P}\left(\mathbf{1}_{E_{c}} \mathbf{1}_{\left\{\left|W_{t}+\hat{z}\right| \mathrm{e}^{-\underline{\lambda}\left|W_{t}+\hat{z}\right|} \geq M\langle k, h\rangle^{-1} \mathrm{e}^{-\underline{\lambda} z}\right\}}\right) \mathrm{d} t-T\right) \\
& \geq \frac{1}{2} \tilde{\pi}_{i}\left(\sum_{k:|k| \geq K^{*}} \hat{p}_{k}(i) \int_{0}^{+\infty} \mathbb{P}\left(\mathbf{1}_{E_{c}} \mathbf{1}_{\left\{1+z \leq\left|W_{t}\right| \leq \log ^{+}\langle k, h\rangle / 2 \underline{\lambda}\right\}}\right) \mathrm{d} t-T\right) \\
& =\frac{1}{2} \tilde{\pi}_{i}\left(\sum_{k:|k| \geq K^{*}} \hat{p}_{k}(i) \mathbb{P}\left(\mathbf{1}_{E_{c}} \int_{0}^{+\infty} \mathbf{1}_{\left\{1+z \leq\left|W_{t}\right| \leq \log ^{+}\langle k, h\rangle / 2 \underline{\lambda}\right\}} \mathrm{d} t\right)-T\right) .
\end{aligned}
$$

Note that $\left(\left|W_{t}\right|, \mathbb{P}\right)$ is a Bessel-3 process starting from 0 . Let $l^{a}, a \geq 0$, be the family of its local times, then the process $\left\{l_{\infty}^{a}, a \geq 0\right\}$ is a $\mathrm{BESQ}^{2}(0)$ process which implies that $l_{\infty}^{a} \stackrel{\mathrm{D}}{=} a l_{\infty}^{1}$ and $\mathbb{P}\left(l_{\infty}^{1}=0\right)=0$ (see [15, Exercise 2.5]). Then we have the following calculations:

$$
\begin{aligned}
& \mathbb{P}\left(\mathbf{1}_{E_{c}} \int_{0}^{+\infty} \mathbf{1}_{\left\{1+z \leq\left|W_{t}\right| \leq \log ^{+}\langle k, h\rangle / 2 \underline{\lambda}\right\}} \mathrm{d} t\right) \\
& \quad=\mathbb{P}\left(\mathbf{1}_{E_{c}} \int_{1+z}^{\log ^{+}\langle k, h\rangle / 2 \underline{\lambda}} l_{\infty}^{a} \mathrm{~d} a\right) \\
&=\mathbb{P}\left(\mathbf{1}_{E_{c}} \int_{1+z}^{\log ^{+}\langle k, h\rangle / 2 \underline{\lambda}} a \mathrm{~d} a \int_{0}^{a^{-1} l_{\infty}^{a}} \mathrm{~d} u\right) \\
&=\int_{1+z}^{\log ^{+}\langle k, h\rangle / 2 \underline{\lambda}} a \mathrm{~d} a \int_{0}^{+\infty} \mathbb{P}\left(\mathbf{1}_{E_{c}} \mathbf{1}_{\left\{u \leq a^{-1} l_{\infty}^{a}\right\}}\right) \mathrm{d} u \\
& \geq \int_{1+z}^{\log ^{+}\langle k, h\rangle / 2 \underline{\lambda}} a \mathrm{~d} a \int_{0}^{+\infty}\left(\mathbb{P}\left(E_{c}\right)-\mathbb{P}\left(a^{-1} l_{\infty}^{a}<u\right)\right)^{+} \mathrm{d} u \\
&= \frac{1}{2}\left(\frac{\log ^{+}\langle k, h\rangle}{2 \underline{\lambda}}-1-z\right)^{2} \int_{0}^{+\infty}\left(\mathbb{P}\left(E_{c}\right)-\mathbb{P}\left(l_{\infty}^{1}<u\right)\right)^{+} \mathrm{d} u .
\end{aligned}
$$

In view of (25) and (26), we obtain

$$
\sum_{k:|k| \geq K^{*}} \hat{p}_{k}(i)\left(\frac{\log ^{+}\langle k, h\rangle}{2 \underline{\lambda}}-1-z\right)^{2} \int_{0}^{+\infty}\left(\mathbb{P}\left(E_{c}\right)-\mathbb{P}\left(l_{\infty}^{1}<u\right)\right)^{+} \mathrm{d} u<+\infty .
$$

Given that

$$
E\left(\xi_{i j}\left(\log ^{+} \xi_{i j}\right)^{2}\right)=+\infty
$$

we have

$$
\sum_{k \in \mathbb{Z}_{+}^{d}} \hat{p}_{k}(i)\left(\log ^{+}\langle k, h\rangle\right)^{2}=+\infty
$$


It follows from (27) that

$$
\int_{0}^{+\infty}\left(\mathbb{P}\left(E_{c}\right)-\mathbb{P}\left(l_{\infty}^{1}<u\right)\right)^{+} \mathrm{d} u=0 .
$$

Thus, by the fact that $\mathbb{P}\left(l_{\infty}^{1}=0\right)=0$, we have $\mathbb{P}\left(E_{c}\right)=0$ for arbitrary $c>0$; consequently, $\widetilde{R}_{0 y}\left(E_{c}\right)=0$ for arbitrary $c>0$. Thus, we complete the proof of part (a).

(b) Choose $\lambda \in(0, \underline{\lambda})$. We have

$$
\begin{aligned}
\sum_{n=0}^{+\infty}(z & \left.+\widetilde{X}\left(\zeta_{\varepsilon_{n}}\right)+\underline{\lambda} \zeta_{\varepsilon_{n}}\right)\left\langle A_{\varepsilon_{n}}, h\right\rangle \mathrm{e}^{-\underline{\lambda}\left(\widetilde{X}\left(\zeta_{\varepsilon_{n}}\right)+\underline{c} \zeta_{\varepsilon_{n}}\right)} \\
& =\sum_{n=0}^{+\infty}(\cdots) \mathbf{1}_{\left\{\left\langle A_{\varepsilon_{n}}, h\right\rangle \leq \mathrm{e}^{\lambda\left(\tilde{X}\left(\zeta \varepsilon_{n}\right)+\underline{c} \zeta \varepsilon_{n}\right)}\right\}}+\sum_{n=0}^{+\infty}(\cdots) \mathbf{1}_{\left\{\left\langle A_{\varepsilon_{n}}, h\right\rangle>\mathrm{e}^{\lambda\left(\tilde{X}\left(\zeta \varepsilon_{n}\right)+\underline{\zeta} \xi_{n}\right)}\right\}} \\
& \triangleq \Theta+\Lambda .
\end{aligned}
$$

We only need to prove that both $\Theta$ and $\Lambda$ are finite almost surely under $\widetilde{R}_{0 y}$.

Hereafter, we write ' $A \lesssim B$ ' to mean that there exists some constant $c>0$ such that $A \leq c B$. Recall that, conditioned on $g^{\widetilde{Y}}$, the split times of the spine is a Poisson point process with characteristic measure $\left(a_{\tilde{Y}_{t}}+\lambda^{*}\right) \mathrm{d} t$. Therefore,

$$
\begin{aligned}
& \widetilde{R}_{0 y}(\Theta)=\widetilde{R}_{0 y}\left(\int_{0}^{+\infty}\left(a_{\widetilde{Y}_{s}}+\lambda^{*}\right)(z+\tilde{X}(s)+\underline{\lambda} s)\left\langle A_{\varepsilon_{n_{s}}}, h\right\rangle\right. \\
& \left.\times \mathrm{e}^{-\underline{\lambda}(\tilde{X}(s)+\underline{c} s)} \mathbf{1}_{\left\{\left\langle A_{\varepsilon_{n_{s}}}, h\right\rangle \leq \mathrm{e}^{\lambda(\tilde{X}(s)+\underline{c s}\}}\right\}} \mathrm{d} s\right) \\
& \leq \int_{0}^{+\infty} \sum_{i \in S}\left(a_{i}+\lambda^{*}\right) \hat{\mathbb{P}}_{y}\left(\tilde{Y}_{s}=i\right) \sum_{k} \hat{p}_{k}(i) \hat{\mathbb{B}} \frac{\lambda}{z}\left(\operatorname{Bes}(s) \mathrm{e}^{-(\underline{\lambda}-\lambda)(\operatorname{Bes}(s)-z)}\right. \\
& \left.\times \mathbf{1}_{\left\{\operatorname{Bes}(s) \geq \lambda^{-1} \log ^{+}\langle k, h\rangle+z\right\}}\right) \mathrm{d} s \\
& \lesssim \sum_{i \in S} \sum_{k} \hat{p}_{k}(i) \int_{0}^{+\infty} \mathbb{P}\left(\left|W_{s}+\hat{z}\right| \mathrm{e}^{-(\underline{\lambda}-\lambda)\left|W_{s}+\hat{z}\right|} \mathbf{1}_{\left\{\left|W_{s}+\hat{z}\right| \geq \lambda^{-1} \log ^{+}\langle k, h\rangle+z\right\}}\right) \mathrm{d} s \\
& \lesssim \sum_{i \in S} \sum_{k} \hat{p}_{k}(i) \int_{\left\{|y+\hat{z}| \geq \lambda^{-1} \log ^{+}\langle k, h\rangle+z\right\}}|y+\hat{z}| \mathrm{e}^{-(\underline{\lambda}-\lambda)|y+\hat{z}|} \mathrm{d} y \\
& \times \int_{0}^{+\infty} s^{-3 / 2} \mathrm{e}^{-|y|^{2} / 2 \pi s} \mathrm{~d} s \\
& \lesssim \sum_{i \in S} \sum_{k} \hat{p}_{k}(i) \int_{\left\{|y+\hat{z}| \geq \lambda^{-1} \log ^{+}\langle k, h\rangle+z\right\}} \frac{|y+\hat{z}|}{|y|} \mathrm{e}^{-(\underline{\lambda}-\lambda)|y+\hat{z}|} \mathrm{d} y \\
& \lesssim \sum_{i \in S} \sum_{k} \hat{p}_{k}(i) \int_{\left\{|y| \geq \lambda^{-1} \log ^{+}\langle k, h\rangle\right\}} \frac{|y|+z}{|y|} \mathrm{e}^{-(\underline{\lambda}-\lambda)|y|} \mathrm{d} y \\
& \lesssim \sum_{i \in S} \sum_{k} \hat{p}_{k}(i) \int_{\lambda^{-1} \log ^{+}\langle k, h\rangle}^{+\infty}\left(r^{2}+z r\right) \mathrm{e}^{-(\underline{\lambda}-\lambda) r} \mathrm{~d} r \\
& <+\infty \text {. }
\end{aligned}
$$

Thus, $\widetilde{R}_{0 y}(\Theta<+\infty)=1$. 
On the other hand, we have

$$
\begin{aligned}
& \widetilde{R}_{0 y}\left(\sum_{n=0}^{+\infty} \mathbf{1}_{\left\{\left\langle A_{\varepsilon_{n}}, h\right\rangle>\mathrm{e}^{\lambda\left(\tilde{X}\left(\zeta_{n}\right)+\underline{c} \zeta \varepsilon_{n}\right)}\right\}}\right) \\
& \quad=\int_{0}^{+\infty} \sum_{i \in S}\left(a_{i}+\lambda^{*}\right) \hat{\mathbb{P}}_{y}\left(\widetilde{Y}_{s}=i\right) \sum_{k} \hat{p}_{k}(i) \hat{\mathbb{B}} \frac{\lambda}{z}\left(\operatorname{Bes}(s)<\lambda^{-1} \log ^{+}\langle k, h\rangle+z\right) \mathrm{d} s,
\end{aligned}
$$

here we have used the fact that the split times of the spine is a Poisson point process with characteristic measure $\left(a_{\widetilde{Y}_{t}}+\lambda^{*}\right) \mathrm{d} t$. Then

$$
\begin{aligned}
\widetilde{R}_{0 y}( & \left.\sum_{n=0}^{+\infty} \mathbf{1}_{\left\{\left\langle A_{\varepsilon_{n}}, h\right\rangle>\mathrm{e}^{\lambda\left(\tilde{X}(\zeta n)+c \xi \xi \varepsilon_{n}\right)}\right\}}\right) \\
& \lesssim \sum_{i \in S} \sum_{k} \hat{p}_{k}(i) \int_{0}^{+\infty} \mathbb{P}\left(\left|W_{s}+\hat{z}\right|<\lambda^{-1} \log ^{+}\langle k, h\rangle+z\right) \mathrm{d} s \\
& \lesssim \sum_{i \in S} \sum_{k} \hat{p}_{k}(i) \int_{\left\{|y+\hat{z}|<\lambda^{-1} \log ^{+}\langle k, h\rangle+z\right\}} \mathrm{d} y \int_{0}^{+\infty} s^{-(3 / 2) \mathrm{e}^{-|y|} / 2 \pi s} \mathrm{~d} s \\
& \leq \sum_{i \in S} \sum_{k} \hat{p}_{k}(i) \int_{\left\{|y|<\lambda^{-1} \log ^{+}\langle k, h\rangle+2 z\right\}}|y|^{-1} \mathrm{~d} y .
\end{aligned}
$$

Consequently, we get

$$
\begin{aligned}
\widetilde{R}_{0 y}\left(\sum_{n=0}^{+\infty} \mathbf{1}_{\left\{\left\langle A_{\varepsilon_{n}}, h\right\rangle>\mathrm{e}^{\lambda\left(\tilde{X}(\zeta n)+\underline{c} \zeta \xi_{n}\right)}\right\}}\right) & \lesssim \sum_{i \in S} \sum_{k} \hat{p}_{k}(i)\left(\lambda^{-1} \log ^{+}\langle k, h\rangle+2 z\right)^{2} \\
& <\infty
\end{aligned}
$$

where in the last inequality we used the condition that $E\left(\xi_{i j}\left(\log ^{+} \xi_{i j}\right)^{2}\right)<+\infty$. Therefore, by (28) we have

$$
\widetilde{R}_{0 y}\left(\sum_{n=0}^{+\infty} \mathbf{1}_{\left\{\left\langle A_{\varepsilon_{n}}, h\right\rangle>\mathrm{e}^{\left.\lambda\left(\widetilde{X}(\zeta n)+\underline{c} \zeta \varepsilon_{n}\right)\right\}}\right.}<+\infty\right)=1,
$$

which means that $\Lambda$ is a finite sum. Hence, $\widetilde{R}_{0 y}(\Lambda<+\infty)=1$. Thus, we complete the proof of part (b).

Proof of Theorem 5. Suppose that $E\left(\xi_{i j}\left(\log ^{+} \xi_{i j}\right)^{2}\right)=+\infty$ for some $i, j \in S$. Since

$$
V_{\underline{\lambda}}\left(\varepsilon_{n}\right) \geq\left\langle A_{\varepsilon_{n}}, b\right\rangle\left(z+\tilde{X}\left(\zeta_{\varepsilon_{n}}\right)+\underline{\lambda} \zeta_{\varepsilon_{n}}\right) \mathrm{e}^{-\underline{\lambda}\left(\tilde{X}\left(\zeta_{\varepsilon_{n}}\right)+\underline{c} \zeta_{\varepsilon_{n}}\right)},
$$

using Lemma 12(a), we have $\widetilde{R}_{0 y}\left(\lim \sup _{t \rightarrow+\infty} V_{\underline{\lambda}}(t)=+\infty\right)=1$. Thus, $P_{0 y}(V(\underline{\lambda})=0)=1$ by Lemma 8 .

On the other hand, suppose that $E\left(\xi_{i j}\left(\log ^{+} \xi_{i j}\right)^{2}\right)<+\infty$ for all $i, j \in S$. Recall that, under $\widetilde{R}_{0 y},\{z+\widetilde{X}(t)+\underline{\lambda} t: t \geq 0\}$ is a Bessel-3 process which is transient, i.e. $\widetilde{R}_{0 y}\left(\lim _{t \rightarrow+\infty}\right.$ $\times(z+\tilde{X}(t)+\underline{\lambda} t)=+\infty)=1$, then from the spine decomposition for $V_{\underline{\lambda}}(t)$ and Lemma 12(b), we have $\widetilde{R}_{0 y}\left(\lim \sup _{t \rightarrow+\infty} \widetilde{R}_{0 y}\left(V_{\underline{\lambda}}(t) \mid \widetilde{G}\right)<+\infty\right)=1$. By Fatou's lemma, we get $\widetilde{R}_{0 y}\left(\lim \sup _{t \rightarrow+\infty} V_{\underline{\lambda}}(t)<+\infty\right)=1$, which implies that $V_{\underline{\lambda}}(t)$ converges to $V(\underline{\lambda})$ in $L^{1}\left(P_{0 y}\right)$. Thus, $P_{0 y}(V(\underline{\lambda})=0)<1$. Similar analysis as in the proof of Theorem 3 can be applied here to show that $P_{0 y}(V(\underline{\lambda})=0)=0$. Hence, we complete the proof. 
Proof of Theorem 2. Using the same techniques as in the supercritical case, we can prove that $\underline{w}(x, y)$ satisfies

$$
\underline{w}(x, y)=E_{0 y}\left[\prod_{u \in Z(s)} \underline{w}\left(x+X_{u}(s)+\underline{c} s, Y_{u}\right)\right],
$$

for any $x \in \mathbb{R}$ and $y \in S$. Obviously, $\lim _{x \rightarrow+\infty} \underline{w}(x, y)=1$ and $\lim _{x \rightarrow-\infty} \underline{w}(x, y)=0$. Thus, $\underline{w}(x, y)$ provides a nontrivial traveling wave solution to (1). Note that $E_{0 y} M(\underline{\lambda})=$ $\lim _{t \rightarrow+\infty} E_{0 y} V_{\underline{\lambda}}(t)=E_{0 y} V_{\underline{\lambda}}(0)=x h_{y}$, and that $\lim _{x \rightarrow+\infty} \underline{w}(x, y)=1$; thus,

$$
\frac{1-\underline{w}(x, y)}{x h_{y} \mathrm{e}^{-\underline{\lambda} x}}=\frac{1-E_{0 y}\left[\exp \left\{-\mathrm{e}^{-\underline{\lambda} x} M(\underline{\lambda})\right\}\right]}{E_{0 y}\left[\mathrm{e}^{-\underline{\lambda} x} M(\underline{\lambda})\right]} \rightarrow 1 \quad \text { as } x \uparrow+\infty .
$$

Next we prove the uniqueness. Consider the space-time barrier $\Gamma^{(z, \underline{\lambda})}$ for $z \geq 0$. By arresting lines of descendants the first time they hit this barrier we again produce a sequence of stopping lines $\{C(z, \underline{\lambda}): z \geq 0\}$ which are dissecting and tending to infinity. Recall that $C(z, \underline{\lambda})=\bigcup_{i \in S} C_{i}\left(z, c_{\lambda}\right)$ (see the proof of Theorem 1(a)). Suppose that $\Phi_{\underline{c}}$ is any traveling wave with speed $\underline{c}$, then

$$
M_{z}(x, \underline{\lambda}):=\prod_{u \in C(z, \underline{\lambda})} \Phi_{\underline{c}}\left(x+X_{u}(t)+\underline{c} t, Y_{u}\right)=\exp \left\{\sum_{i \in S} \sharp C_{i}(z, \underline{\lambda}) \log \Phi_{\underline{c}}(x+z, i)\right\}
$$

is a $P_{0 y}$-martingale which converges to $\Phi_{\underline{c}}(x, y)$ almost surely and in $L^{1}\left(P_{0 y}\right)$.

We turn our attention to the MBBM with a killing barrier at $\Gamma^{(-x, \underline{\lambda})}$ where $x>0$. Define $\tilde{C}(z, \underline{\lambda})$ to be the random set of particles for the killed process that are stopped at the barrier $\Gamma^{(z, \underline{\lambda})}$. More precisely, $\tilde{C}(z, \underline{\lambda}):=\bigcup_{i \in S} \tilde{C}_{i}(z, \underline{\lambda})$ consists of particles whose lines of descendants (including themselves) have spatial paths that have met the barrier $\Gamma^{(z, \underline{\lambda})}$ before meeting $\Gamma^{(-x, \underline{\lambda})}$, where $\tilde{C}_{i}(z, \underline{\lambda})$ denotes the subset of type $i$ particles. Recall that $\gamma^{(-x, \underline{\lambda})}$ denotes the event that the MBBM remains entirely to the right of $\Gamma^{(-x, \underline{\lambda})}$ and $P_{0 y}\left(\gamma^{(-x, \underline{\lambda})}\right) \uparrow 1$ as $x \uparrow+\infty$. On the event $\gamma_{\widetilde{C}_{i}}^{(-x, \underline{\lambda})}$ the MBBM and the MBBM with killing barrier $\Gamma^{(-x, \underline{\lambda})}$ are the same, i.e. $\sharp C_{i}(z, \underline{\lambda})=\sharp \widetilde{C}_{i}(z, \underline{\lambda})$ on $\gamma^{(-x, \underline{\lambda})}$. Therefore,

$$
\lim _{z \rightarrow+\infty}-\sum_{i \in S} \sharp \tilde{C}_{i}(z, \underline{\lambda}) \log \Phi_{\underline{c}}(z, i)
$$

exists almost surely and is nonnegative on $\gamma^{(-x, \underline{\lambda})}$. Furthermore, since the function $x \mapsto$ $\Phi_{\underline{c}}(x, y)$ is nontrivial, an elementary argument shows that, for $x>0$ sufficiently large, $\lim _{z \rightarrow+\infty}-\sum_{i \in S} \sharp \tilde{C}_{i}(z, \underline{\lambda}) \log \Phi_{\underline{c}}(z, i)$ is positive with positive probability on $\gamma^{(-x, \underline{\lambda})}$. Consider the sequence

$$
\begin{aligned}
V_{\Gamma^{(z, \underline{\lambda})}}^{x} & :=\left(h_{y} x\right)^{-1} \sum_{u \in \tilde{C}(z, \underline{\lambda})} h_{Y_{u}}\left(x+X_{u}(t)+\underline{\lambda} t\right) \mathrm{e}^{-\underline{\lambda}\left(X_{u}(t)+\underline{c} t\right)} \\
& =\left(h_{y} x\right)^{-1}(x+z) \sum_{i \in S} h_{i} \sharp \tilde{C}_{i}(z, \underline{\lambda}) \mathrm{e}^{-\underline{\lambda} z} .
\end{aligned}
$$

Let $\widetilde{\mathcal{F}}_{\Gamma^{\left(z, c_{\lambda}\right)}}$ be the natural filtration generated by ancestral type and spatial paths receding from particles at the moment they hit $\Gamma^{\left(z, c_{\lambda}\right)}$ before meeting $\Gamma^{(-x, \underline{\lambda})}$. By the property of dissecting 
stopping lines, $\left\{V_{\Gamma^{(z, \lambda)}}^{x}: z \geq 0\right\}$ is a mean-one $P_{0 y}$-martingale with respect to $\left\{\widetilde{\mathcal{F}}_{\Gamma^{\left(z, c_{\lambda}\right)}}: z \geq 0\right\}$, and

$$
\lim _{z \rightarrow+\infty}(x+z) \mathrm{e}^{-\underline{\lambda} z} \sum_{i \in S} \sharp \tilde{C}_{i}(z, \underline{\lambda}) h_{i}=M(\underline{\lambda}), \quad P_{0 y} \text {-almost surely. }
$$

The arguments on $W_{\Gamma^{(x, \lambda)}}(\lambda)$ in the proof of Theorem 1 still work when $\lambda=\underline{\lambda}$; thus, we have

$$
\lim _{z \rightarrow+\infty} \sum_{i \in S} \sharp \tilde{C}_{i}(z, \underline{\lambda}) h_{i} \mathrm{e}^{-\underline{\lambda} z}=0, \quad P_{0 y} \text {-almost surely. }
$$

Combining (30) and (31), we obtain

$$
\lim _{z \rightarrow+\infty} z \mathrm{e}^{-\underline{\lambda} z} \sum_{i \in S} \sharp \tilde{C}_{i}(z, \underline{\lambda}) h_{i}=M(\underline{\lambda}), \quad P_{0 y} \text {-almost surely. }
$$

Applying similar arguments as in the supercritical case, we know that $\left\{\left(\sharp C_{1}(z, \underline{\lambda}), \ldots\right.\right.$, $\left.\sharp C_{d}(z, \underline{\lambda})\right)^{\top}: z \geq 0$ \} forms a nonextinct, positive regular continuous time multitype Markov branching process ( $z$ plays the role of time). By the Kesten-Stigum theorem (see, for example, [6, Theorem 2.1]), there is a nonnegative vector $\pi_{\underline{\lambda}}=\left(\pi_{\underline{\lambda}}^{1}, \ldots, \pi_{\underline{\lambda}}^{d}\right)^{\top}$ such that $\left\langle\pi_{\underline{\lambda}}, 1\right\rangle=1$ and, for all $i \in S, P_{0 y}\left(\lim _{z \rightarrow+\infty} \sharp C_{i}(z, \underline{\lambda}) / \sharp C(z, \underline{\lambda})=\pi_{\lambda}^{i}\right) \stackrel{\underline{\lambda}}{=} 1$ and, consequently, $\lim _{z \rightarrow+\infty}$ $\sharp \tilde{C}_{i}(z, \underline{\lambda}) / \sharp \tilde{C}(z, \underline{\lambda})=\pi_{\underline{\lambda}}^{i}$ almost surely on $\gamma^{(-x, \underline{\lambda})}$. Let $x^{\underline{\lambda}}+\infty$, we have

$$
\lim _{z \rightarrow+\infty} \frac{\sharp \tilde{C}_{i}(z, \underline{\lambda})}{\sharp \tilde{C}(z, \underline{\lambda})}=\pi_{\underline{\lambda}}^{i}, \quad P_{0 y} \text {-almost surely. }
$$

Let $\tilde{\pi}=\pi_{\underline{\lambda}} /\langle h, \pi\rangle$. Using (32), (33), and the fact that $h_{i}>0$ for every $i \in S$, we get that, for all $i \in S$,

$$
\lim _{z \rightarrow+\infty} z \mathrm{e}^{-\underline{\lambda} z} \sharp \tilde{C}_{i}(z, \underline{\lambda})=\tilde{\pi}_{i} M(\underline{\lambda}), \quad P_{0 y} \text {-almost surely. }
$$

From (29) and (34), we conclude that $\beta:=\lim _{z \rightarrow+\infty}-z^{-1} \mathrm{e}^{\underline{\lambda} z} \sum_{i \in S} \tilde{\pi}_{i} \log \Phi_{\underline{c}}(z, i)$ exists and is positive. Uniqueness (up to a multiplicative constant) is now immediate. In fact,

$$
\begin{aligned}
\Phi_{\underline{c}}(x, y) & =E_{0 y}\left(\lim _{z \rightarrow+\infty} M_{z}(x, \underline{\lambda})\right) \\
& =E_{0 y} \exp \left\{\lim _{z \rightarrow+\infty} \sum_{i \in S} \sharp C_{i}(z, \underline{\lambda}) \log \Phi_{\underline{c}}(x+z, i)\right\} .
\end{aligned}
$$

Since $\lim _{\eta \uparrow+\infty} P_{0 y}\left(\gamma^{(-\eta, \underline{\lambda})}\right)=1$, using (34) we obtain

$$
\begin{aligned}
\Phi_{\underline{c}}(x, y)= & \lim _{\eta \uparrow+\infty} E_{0 y}\left[\exp \left\{\lim _{z \rightarrow+\infty} \sum_{i \in S} \sharp \tilde{C}_{i}(z, \underline{\lambda}) \log \Phi_{\underline{c}}(x+z, i)\right\} ; \gamma^{(-\eta, \underline{\lambda})}\right] \\
= & \lim _{\eta \uparrow+\infty} E_{0 y}\left[\exp \left\{\lim _{z \rightarrow+\infty} \sum_{i \in S} \tilde{\pi}_{i} M(\underline{\lambda}) \mathrm{e}^{\underline{\lambda} z} z^{-1} \log \Phi_{\underline{c}}(x+z, i)\right\} ; \gamma^{(-\eta, \underline{\lambda})}\right] \\
= & E_{0 y} \exp \left\{-M(\underline{\lambda}) \mathrm{e}^{-\underline{\lambda} x}\right. \\
& \left.\times \lim _{z \rightarrow+\infty}-\frac{x+z}{z} \sum_{i \in S} \tilde{\pi}_{i}(x+z)^{-1} \mathrm{e}^{\underline{\lambda}(x+z)} \log \Phi_{\underline{c}}(x+z, i)\right\} \\
= & E_{0 y} \exp \left\{-\beta M(\underline{\lambda}) \mathrm{e}^{-\underline{\lambda} x}\right\} .
\end{aligned}
$$

Hence, we complete the proof. 


\section{Acknowledgement}

The authors sincerely thank an anonymous referee for helpful comments.

\section{References}

[1] Athreya, K. B. (1968). Some results on multitype continuous time Markov branching processes. Ann. Math. Statist. 39, 347-357.

[2] Athreya, K. B. ANd Ney, P. E. (2004). Branching Processes. Dover, Mineola, NY.

[3] Champneys, A. et al. (1995). Algebra, analysis and probability for a coupled system of reaction-diffusion equations. Philos. Trans. R. Soc. London Ser. A 350, 69-112.

[4] Chauvin, B. and Rouault, A. (1988). KPP equation and supercritical branching Brownian motion in the subcritical speed area. Application to spatial trees. Prob. Theory Relat. Fields 80, 299-314.

[5] Durrett, R. (1996). Probability: Theory and Examples, 2nd edn. Duxbury Press, Belmont, CA.

[6] Georgit, H.-O. and BaAKe, E. (2003). Supercritical multitype branching processes: the ancestral types of typical individuals. Adv. Appl. Prob. 35, 1090-1110.

[7] Hardy, R. And Harris, S. C. (2009). A spine approach to branching diffusions with applications to $L^{p}$ convergence of martingales. In Séminaire de Probabilités XLII (Lecture Notes Math. 1979), Springer, Berlin, pp. 281-330.

[8] Harris, S. C. (1999). Traveling-waves for the FKPP equation via probabilistic arguments. Proc. R. Soc. Edinburgh Sect. A 129, 503-517.

[9] Jagers, P. (1989). General branching processes as Markov fields. Stoch. Process. Appl. 32, 183-212.

[10] Kurtz, T., Lyons, R., Pemantle, R. and Peres, Y. (1997). A conceptual proof of the Kesten-Stigum theorem for multi-type branching processes. In Classical and Modern Branching Processes, Springer, New York, pp. 181-185.

[11] Kyprianou, A. E. (2004). Travelling wave solutions to the K-P-P equation: alternatives to Simon Harris' probabilistic analysis. Ann. Inst. H. Poincaré Prob. Statist. 40, 53-72.

[12] Kyprianou, A. E., Liu, R.-L., Murillo-Salas, A. and Ren, Y.-X. (2012). Supercritical super-Brownian motion with a general branching mechanism and travelling waves. Ann. Inst. H. Poincaré Prob. Statist. 48, 661-687.

[13] LyONS, R. (1997). A simple path to Biggins' martingale convergence for branching random walk. In Classical and Modern Branching Processes, Springer, New York, pp. 217-221.

[14] McKean, H. P. (1975). Application of Brownian motion to the equation of Kolmogorov-Petrovskii-Piskunov. Commun. Pure Appl. Math. 28, 323-331. (Correction: 29 (1976), 553-554.)

[15] Revuz, D. AND Yor, M. (1991). Continuous Martingales and Brownian Motion. Springer, Berlin.

[16] Seneta, E. (1973). Non-Negative Matrices. An Introduction to Theory and Applications. Halsted Press, New York. 